\title{
A Unified Distributed Cooperative Control of DC Microgrids Using Consensus Protocol
}

\author{
Li, Yu; Zhang, Zhenbin; Dragicevic, Tomislav; Rodriguez, Jose
}

Published in:

IEEE Transactions on Smart Grid

Link to article, DOI:

10.1109/TSG.2020.3041378

Publication date:

2021

Document Version

Peer reviewed version

Link back to DTU Orbit

Citation (APA):

Li, Y., Zhang, Z., Dragicevic, T., \& Rodriguez, J. (2021). A Unified Distributed Cooperative Control of DC Microgrids Using Consensus Protocol. IEEE Transactions on Smart Grid, 12(3), 1880 - 1892.

https://doi.org/10.1109/TSG.2020.3041378

\section{General rights}

Copyright and moral rights for the publications made accessible in the public portal are retained by the authors and/or other copyright owners and it is a condition of accessing publications that users recognise and abide by the legal requirements associated with these rights.

- Users may download and print one copy of any publication from the public portal for the purpose of private study or research.

- You may not further distribute the material or use it for any profit-making activity or commercial gain

- You may freely distribute the URL identifying the publication in the public portal

If you believe that this document breaches copyright please contact us providing details, and we will remove access to the work immediately and investigate your claim. 


\title{
A Unified Distributed Cooperative Control of DC Microgrids Using Consensus Protocol
}

\author{
Yu Li, Student Member, IEEE, Zhenbin Zhang, Senior Member, IEEE, \\ Tomislav Dragičević Senior Member, IEEE, and Jose Rodriguez, Fellow, IEEE
}

\begin{abstract}
In this work, we propose an effective and simple control approach for islanded DC microgrids that allows each distributed generator (DG) to achieve accurate voltage regulation and power-sharing. An improved dynamic consensus protocol, which is robust to measurement noise and states initialization, is employed to enable each agent to locally calculate the average bus voltage with a sparse cyber network. On this basis, we propose a cooperative controller that merges the voltage regulation and power-sharing objectives in a unified fashion. The proposed approach only uses neighbors' voltage information to regulates the average bus voltage to its nominal value while maintaining proportional power-sharing or optimal power dispatch. This significantly simplifies its implementation and reduces the communication bandwidth requirement. A global model of the DC microgrid considering the cyber network is established in the form of a state-space-model, where the reference voltage vector corresponds to the input and the average bus voltage vector denotes the state. Then, the input-to-state stability analysis is carried out. To the end, comprehensive hardware-in-the-loop (HiL) tests are conducted to validate the effectiveness of the proposed control strategy. The proposed control strategy exhibits plug-and-play capability, and it is resilient to message update rate and communication failure.
\end{abstract}

Index Terms-Cooperative control, DC microgrids, distributed control, load sharing, stability analysis

\section{INTRODUCTION}

$\mathbf{N}$ OWADAYS, with the large scale penetration of distributed generations (DGs), the power system is developing rapidly towards a more distributed and more powerelectronics-based paradigm [1]. To fully exploit the potential of DGs and realize the reliable energy supply to the load, microgrids are regarded as the most promising solution [2][5]. According to their operation forms, microgrids can be divided into three categories, i.e., alternate current (AC), direct current (DC), and AC-DC Hybrid microgrids [6]. Recently, considering the DC nature of DGs and modern loads, the DC

This work is financially supported in part by the Shandong Provincial Key Research and Development Program (Major Scientific and Technological Innovation Project) (NO.2019JZZY020805), in part by the National Distinguished Expert (Youth Talent) Program of China (31390089963058), in part by the General Program of National Natural Science Foundation of China (51977124), in part by the Shandong Natural Science Foundation (ZR2019QEE001), and in part by the Natural Science Foundation of Jiangsu Province (BK20190204). (Corresponding author: Zhenbin Zhang).

$\mathrm{Y}$. Li and Z. Zhang are with the School of Electrical Engineering, Shandong University, Jinan 250061, China (email: yu.li@mail.sdu.edu.cn, zbz@sdu.edu.cn).

T. Dragičević is with the Center of Electric Power and Energy, Technical University of Denmark, 2800 Kongens Lyngby, Denmark (e-mail: tomdr@elektro.dtu.dk).

J. Rodriguez is with Facultad de Ingenieria, Universidad Andres Bello, Santiago 8370146, Chile (e-mail: jose.rodriguez@unab.cl).

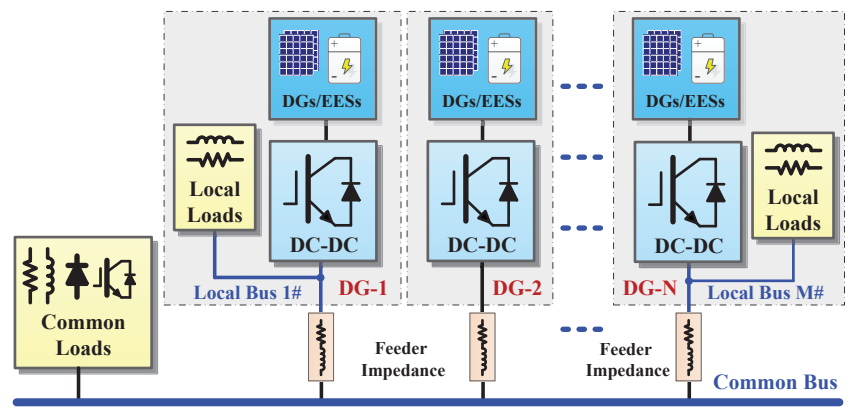

Fig. 1. The general architecture of a multiple buses islanded DC microgrid with heterogeneous DGs and energy storage equipment.

microgrids that allow the system to have fewer conversion stages are gaining more attention. Moreover, the absence of concepts of frequency synchronization and reactive power in a DC system significantly simplifies its operation and control.

A DC microgrid is capable of providing energy for local loads with multiple buses through heterogeneous DGs and energy storage equipment [7], in islanded mode (see Fig. 1). Several critical challenges arise for its efficient and reliable operation [8]. Specifically, (i) high-quality voltage regulation, (ii) the proportional power-sharing or optimal power dispatch and, (iii) scalability and plug-and-play ( $\mathrm{PnP}$ ) capability are essential. To address the above challenges, a variety of control strategies have been reported. To date, using communication while retaining a distributed architecture is considered as a key technique [9]. Recent works in this field are remarkably effective. In [10], the average voltage and current regulators are added on a basic primary controller to achieve powersharing and voltage restoration simultaneously. Accordingly, this method can be categorized as voltage-shifting method. A hybrid method with both voltage-shifting and droop slopeadjusting approach is proposed in [11], which improves the dynamic load sharing performance. However, both methods require each agent to collect information from all the other nodes to obtain the average voltage and current. This is known as broadcast Gossip algorithm in communications [12].

Alternatively, a sparse communication network is adopted using the dynamic consensus algorithm (see more details in Section III) in [13]. A voltage regulator is introduced to correct the voltage deviation, meanwhile a current regulator is applied to ensure the proportional load sharing. Only neighbors' data is used for its implementation. This cooperative control shows its excellent autonomy and adaptability characteristics. However, the parameter tuning process is relatively complex, and the 
TABLE I

KEY FEATURES OF DIFFERENT CONTROL STRATEGY

\begin{tabular}{cccc}
\hline & $\begin{array}{c}\text { voltage } \\
\text { regulation }\end{array}$ & $\begin{array}{c}\text { power } \\
\text { sharing }\end{array}$ & data exchange \\
\hline Nasirian et al. [13] & $\begin{array}{c}\text { voltage } \\
\text { regulator }\end{array}$ & $\begin{array}{c}\text { current } \\
\text { regulator }\end{array}$ & $\begin{array}{c}\text { estimated voltage } \\
\text { \& measured current }\end{array}$ \\
\hline Hamad et al. [14] & $\begin{array}{c}\text { restoring } \\
\text { voltage term }\end{array}$ & $\begin{array}{c}\text { power } \\
\text { sharing term }\end{array}$ & $\begin{array}{c}\text { estimated voltage } \\
\text { \& estimated power }\end{array}$ \\
\hline Trip et al. $[16]$ & \multicolumn{2}{c}{ a unified controller } & measured current \\
\hline $\begin{array}{c}\text { The proposed } \\
\text { control strategy }\end{array}$ & a unified controller & estimated voltage \\
\hline
\end{tabular}

${ }^{1}$ Assume the rated power of each DG is constant during operation.

large-signal stability analysis is not conducted. A multiagent supervisory control for precise power management in isolated dc microgrids is presented in [14]. The average consensus theory is employed along with two power management strategies, achieving power-sharing and optimal power dispatch, respectively. In [15], a consensus-based algorithm is presented to achieve proportional power-sharing and regulation of weighted geometric mean of bus voltage in DC microgrids. The load sharing error and voltage regulation error are derived and maneuvered to zero to realize the above two control objectives. A sufficient condition that stabilizes the system with ZIP loads (constant impedance, constant current, and constant power loads) is established. However, the important performance metrics of the proposed method, e.g. PnP capability and resiliency to communication failure, are not comprehensively investigated.

In summary, all the above methods use two separate controllers to generate two compensation terms to address the voltage deviation and power-sharing inaccuracy, respectively. Although these methods have an intuitive physical concept, the controller parameters design and tuning processes are complex. Furthermore, both voltage and current information from its nearest-neighbor are shared through the communication network resulting in high data throughput. To that end, an effective and simple controller with low communication bandwidth is desired which potentially enhances system reliability. In [16], a distributed averaging control scheme achieves current sharing and average voltage regulation requiring only the measurements of the generated current. Thus, the voltage observer is omitted. This control approach is also independent of the microgrid parameters and the topology of the communication network. However, extending the control scheme to different converter types, such as boost converters, needs to be further investigated.

Motivated by the above observation, we propose a simple distributed control strategy that enables each DG to achieve accurate voltage regulation and power-sharing. The proposed approach merges the voltage regulation and power-sharing objectives in a unified controller, and its optimal parameters are extremely straightforward to obtain. To highlight the salience of this work, a comparison between the proposed approach and state-of-the-art methods are conducted in TABLEI. The main contributions of this work are summarized as below.
1) A new cooperative control framework that requires only the neighborhood voltage information (current knowledge is unnecessary) is proposed, reducing communication and measurement dependency. The proposed controller is independent of the line impedance of the microgrid and it is able to simultaneously regulate the voltage and powersharing. Moreover, the proposed method, as a framework, allows the system to incorporate different power management strategies flexibly (e.g., optimal power dispatch functionality).

2) The proposed observer is insensitive to the state-variable initialization and is robust to measurement noises, which are discussed in detail. On this basis, the plug-and-play capability of the proposed solution is realized, which permits flexible departure or return of any agents associated with the system. The global DC microgrid model is built considering the communication network. To this end, the input-to-state stability analysis of the DC microgrid is conducted, proving the correctness of the proposed cooperative control.

3) A full state-variable Finite Control Set Model Predictive Control (FCS-MPC), as the inner controller, is proposed, which enables the output voltage to track its reference rapidly.

4) The proposed cooperative control is comprehensively validated for different scenarios via a lab-built real-time hardware-in-the-loop (HiL) test-bench. Primary implementation guidelines are provided and discussed as well.

The remaining of this paper is organized as follows. One of the state-of-the-art cooperative control for DC microgrids is reviewed in Section II. Section III describes the dynamic consensus problem in DC microgrids. Section IV presents the proposed distributed controller. The global modeling and stability analysis is conducted in Section V. Section VI evaluates the performance of the proposed scheme through the HiL results. Section VII concludes this work.

\section{State of the Art of Cooperative Control in DC MICROGRIDS}

To facilitate the comparative study, one of the state-of-the art control method in DC microgrids is briefly reviewed in this section. In [13], a cooperative control scheme that only shares information with its immediate neighbors was developed. First, a voltage observer is introduced to estimate the global average voltage. Then, the voltage set point is generated by a voltage regulator and a current regulator. Specifically, the voltage regulator generates a voltage correction term to restore the voltage deviation. Meanwhile, the current regulator compares the local per-unit current of each converter with its neighbors'. Accordingly, this current regulator provides a second voltage correction term to synchronize per-unit currents and, thus, guarantees proportional power-sharing. Fig. 2 shows the proposed distributed cooperative control in [13].

The local voltage set point $v_{i}^{*}$ is

$$
v_{i}^{*}=v_{\mathrm{dc}}^{*}-R_{i}^{o} i_{i}^{o}+\underbrace{H_{i}(s)}_{\left(k_{p v}+\frac{k_{i v}}{s}\right)}\left(v_{\mathrm{dc}}^{*}-\bar{v}_{i}^{\text {bus }}\right)+\underbrace{G_{i}(s)}_{\left(k_{p c}+\frac{k_{i c}}{s}\right)} \delta_{i}
$$




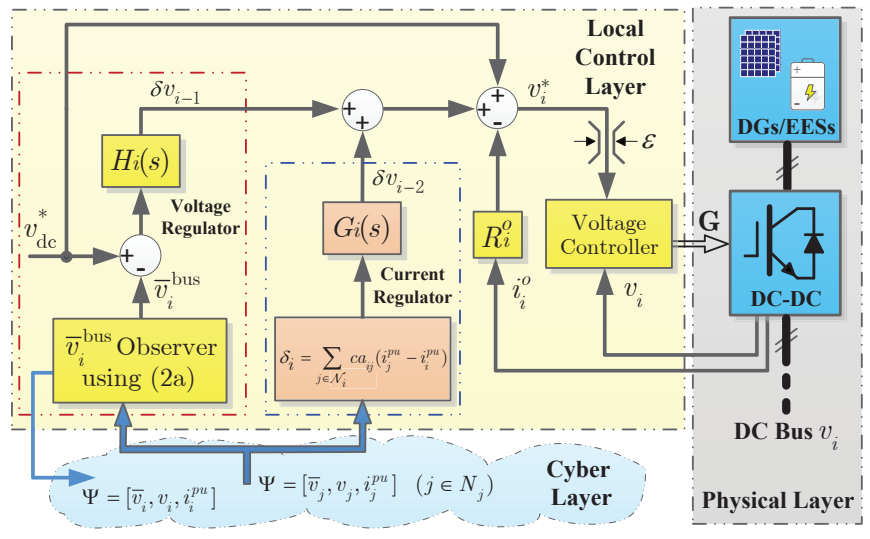

Fig. 2. Distributed cooperative control proposed in [13].

where

$$
\begin{aligned}
\bar{v}_{i}^{\text {bus }}(t) & =v_{i}(t)+\int_{0}^{t} \sum_{j \in \mathcal{N}_{i}} a_{i j}\left(\bar{v}_{j}^{\text {bus }}(\tau)-\bar{v}_{i}^{\text {bus }}(\tau)\right) \mathrm{d} \tau, \\
\delta_{i} & =\sum_{j \in \mathcal{N}_{i}} c a_{i j}\left(i_{j}^{\text {pu }}-i_{i}^{\text {pu }}\right) .
\end{aligned}
$$

$H_{i}(s)$ and $G_{i}(s)$ are PI controllers for voltage restoration and power-sharing, respectively. $k_{p v}, k_{i v}, k_{p c}, k_{i c}$ are corresponding coefficients of PI controllers. $a_{i j}$ is the $i, j$ element of adjacency matrix, and $\mathcal{N}_{i}$ is the set containing all neighbors of node $i$. These definitions will be further clarified in the subsequent section. $c$ is a coupling coefficient, whereas $i_{j}^{p u}$ and $i_{i}^{p u}$ are per-unit current of $i$ and $j$ node, respectively.

This distributed method uses a sparse communication network for data exchange, and shows superior expandability and PnP capability. However, there are two separate controllers need to be properly designed, and considerable tuning effort is mandatory to maximize system performance. Moreover, for each converter, both estimated voltages and local measured currents are shared with its neighbors. This potentially implies high communication bandwidth requirement.

\section{Dynamic Consensus Problem in DC Microgrids}

Consensus problem has attracted significant attention in the control community. The insight of this problem is that several spatially distributed agents must reach a common output value without recourse to a central controller or global communication. The distributed control of microgrids inherently requires tight coordination among DGs in a dynamic fashion, which is a suitable study case for the dynamic consensus algorithm. This section aims to provide a brief overview of the dynamic consensus problem in DC microgrids.

\section{A. Notation of Graph Theory}

As shown in Fig. 3, an undirected (bidirectional) graph with $N$ nodes can be illustrated as $\mathcal{G}=(\mathcal{V}, \mathcal{E})$, where $\mathcal{V}=\{1, \ldots, N\}$ is the agent set and $\mathcal{E} \subseteq \mathcal{V} \times \mathcal{V}$ is the edge set [17]. An edge is $(i, j) \in \mathcal{E}$, only if agent node $j$ sends information to node $i$. Considering the interested graph is undirected, $(i, j) \in \mathcal{E} \Leftrightarrow(j, i) \in \mathcal{E}$ holds. The associated

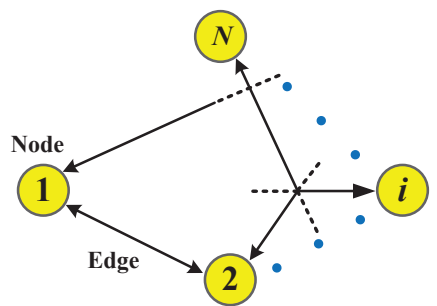

Fig. 3. An undirected graph with $N$ nodes.

weighted adjacency matrix $\boldsymbol{A}_{G}=\left[a_{i j}\right]$ of graph $\mathcal{G}$ is an $N \times N$ matrix where $a_{i j}>0$ if $(i, j) \in \mathcal{E}$, and $a_{i j}=0$, otherwise. $\mathcal{N}_{i}=\{j \mid(i, j) \in \mathcal{E}\}$ denotes the set of all neighbors of node $i$. The weighted degree of node $i$ is, $d_{i}=\sum_{j=1}^{N} a_{i j}$. Finally, the Laplacian matrix is constructed from the adjacency matrix and degree matrix as follows

$$
\boldsymbol{L}=\boldsymbol{D}-\boldsymbol{A}_{G}
$$

\section{B. Dynamic Average Consensus Problem}

Consider a group with $N$ agents where each agent is able to (i) send and receive information with its neighbors, (ii) and perform local status updating using local measurements and shared information. The variable $r_{i}(t)$ is referred to as the reference signal (or input) of node $i$ at time $t$. The average value of the reference inputs from all agents is denoted as

$$
\bar{r}(t)=\frac{1}{N} \sum_{i=1}^{N} r_{i}(t)
$$

$\bar{r}(t)$ is called dynamic average of the reference signals since $r_{i}(t)$ are time-varying variables.

The classical algorithm for dynamic consensus is [18]

$$
\dot{x}_{i}(t)=-\sum_{j \in \mathcal{N}_{i}} a_{i j}\left(x_{i}(t)-x_{j}(t)\right)+\dot{r}_{i}(t), i \in\{1,2, \ldots N\},
$$

which is the differential form of (2a). To obtain the global format, let $\boldsymbol{x}=\left[x_{1}, x_{2}, \ldots, x_{N}\right]^{\top}$ and $\boldsymbol{r}=\left[r_{1}, r_{2}, \ldots, r_{N}\right]^{\top}$ be the state and input vectors, respectively. We have

$$
\dot{\boldsymbol{x}}(t)=-\boldsymbol{L} \boldsymbol{x}(t)+\dot{\boldsymbol{r}}(t) .
$$

\section{Dynamic Average Consensus in DC Microgrids}

As stated, the dynamic average consensus algorithm should be completely distributed, where each agent only obtains information from its neighbors. This feature perfectly fits the demand for a sparse communication network in microgrids. Based on the above interpretation, we let the observed global average bus voltage $\bar{v}_{i}^{\text {bus }}(t)$ of node $i$ match the consensus state $x_{i}(t)$, while local output voltage $v_{i}(t)$ of node $i$ corresponds to the input signal $r_{i}(t)$.

Indeed, the most desired scenario is that all bus voltages are regulated to the nominal value. However, this contradicts the power-sharing requirement because there are voltage drops in the power lines between the DGs (see Fig. 1). Therefore, weighted average bus voltages [19] or weighted geometric 
mean of the bus voltages [15], can be used as alternatives to achieve global voltage regulation. Herein, the observed average bus voltage as the regulation target is directly adopted. Note that other regulation targets are also compatible with the proposed approach.

Specifically, in a DC microgrid with complicated connections (see Fig. 1), a properly designed dynamic average consensus algorithm will ensure the global average bus voltage $\bar{v}_{i}^{\text {bus }}$ converge to $\frac{1}{N} \sum_{i=1}^{N} v_{i}(t)$. Consequently, each DG shares the global information of average bus voltage. To this end, the ultimate goal of controlling a DC microgrid is to regulate the global average voltage to the nominal value while ensuring proportional power-sharing between DGs.

\section{Improved Dynamic Consensus Algorithm}

Although the classical dynamic consensus algorithm (5) or (6) is effective for cooperative control in microgrids, two major concerns are still not fully addressed from the implementation perspective. (i) One concern is that it requires the explicit derivative of the measurements which is sensitive to the noise. (ii) The other one is the initialization condition of the consensus state should satisfy $\sum_{i=1}^{N} \bar{v}_{i}^{\text {bus }}\left(t_{0}\right)=\sum_{i=1}^{N} v_{i}\left(t_{0}\right)$. Nevertheless, the arrival and departure of an agent will break this condition if no re-initialization is implemented.

The first concern can be resolved by a simple algebraic manipulation, i.e., defining a new internal variable $p_{i}=v_{i}-\bar{v}_{i}^{\text {bus }}$, for $i \in\{1, \ldots N\}$. In this way, the derivative of the measured input signal is eliminated. However, the arrival and departure of an agent still limits the implementation of the algorithm. To overcome the initialization problem, herein, an improved dynamic consensus algorithm [17] is introduced

$$
\begin{aligned}
\bar{v}_{i}^{\text {bus }} & =p_{i}+v_{i}, \\
\dot{p}_{i} & =-\alpha p_{i}-\sum_{j \in \mathcal{N}_{i}} a_{i j}\left(\bar{v}_{i}^{\text {bus }}-\bar{v}_{j}^{\text {bus }}\right)+\sum_{j \in \mathcal{N}_{i}} b_{j i}\left(q_{i}-q_{j}\right), \\
\dot{q}_{i} & =-\sum_{j \in \mathcal{N}_{i}} b_{i j}\left(\bar{v}_{i}^{\text {bus }}-\bar{v}_{j}^{\text {bus }}\right),
\end{aligned}
$$

where $\alpha \geq 0$ is a global observer parameter. As observed from (7), an integral term, $q$, and two adjacency matrices, e.g. $\boldsymbol{L}_{\mathrm{P}}=\left[a_{i j}\right]_{N \times N}$ and $\boldsymbol{L}_{\mathrm{I}}=\left[b_{i j}\right]_{N \times N}$, are introduced. In contrast to (5), the additional integral term provides robustness to the state initialization. In particular, $\bar{v}_{i}^{\text {bus }}$ converges to $\frac{1}{N} \sum_{i=1}^{N} v_{i}$ exponentially with arbitrary initial states $x_{i}\left(t_{0}\right)$ and $q_{i}\left(t_{0}\right)$, for all $i \in\{1, \ldots N\}$. The rigorous proof of this conclusion can be found in [20].

\section{UNIFIED Distributed COOPERATIVE CONTROL}

In this section, we develop an effective distributed cooperative control approach to achieve bus voltage regulation and power-sharing simultaneously. The proposed algorithm embeds the above two control objectives into a unified controller.

\section{A. Voltage Regulation and power-sharing}

The proposed approach consists of two main parts, i.e., a voltage regulator and a bus voltage observer, see Fig. 4. Herein, the former is explained in detail, and the latter will be carefully

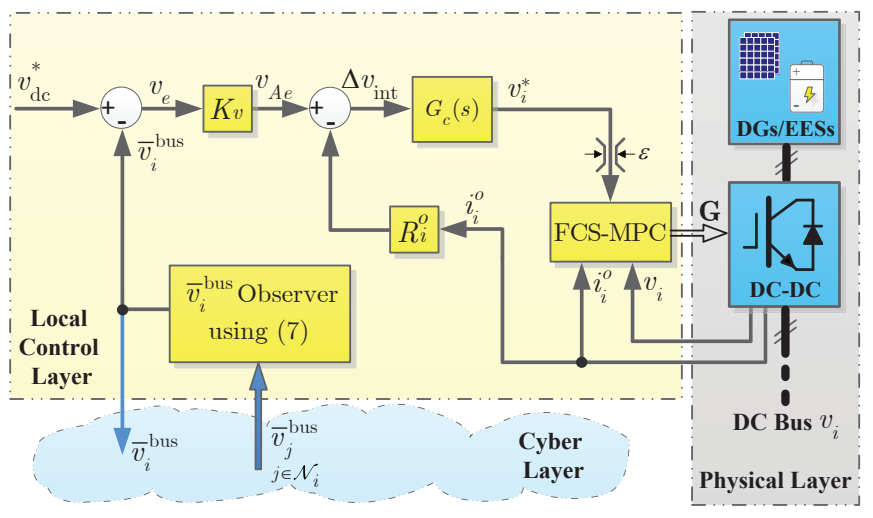

Fig. 4. The block diagram of the proposed unified distributed cooperative control.

discussed in the subsequent subsection. As observed in Fig. 3, firstly, the error $v_{e}$ between nominal bus voltage $v_{\mathrm{dc}}^{*}$ and the estimated average bus voltage $\bar{v}_{i}^{\text {bus }}$ at node $i$ is amplified by $K_{v}$, i.e.

$$
v_{A e}=K_{v}\left(v_{\mathrm{dc}}^{*}-\bar{v}_{i}^{\text {bus }}\right) .
$$

Then, $\Delta v_{\text {int }}$, i.e. the difference between $v_{A e}$ and the voltage drop on the (internal) virtual resistance $i_{i}^{o} \cdot R_{i}^{o}$, is sent into $G_{c}(s)$. Herein, we design a simple compensator as

$$
G_{c}(s)=\frac{1}{s+\alpha},
$$

where the global observer parameter $0<\alpha \ll 1$ holds to make sure the dc gain of $G_{c}(s)$ is considerably large. This entails the frequency characteristic of $G_{c}(s)$ similar to an integrator, while guarantees all poles of the system are in the left halfplane. To this end, the dynamics of voltage reference value $v_{i}^{*}$ is obtained, i.e.,

$$
\dot{v}_{i}^{*}=-\alpha v_{i}^{*}+\left(v_{A e}-i_{i}^{o} \cdot R_{i}^{o}\right) \approx\left(v_{A e}-i_{i}^{o} \cdot R_{i}^{o}\right) .
$$

From $v_{i}^{*}$ to $v_{i}$, a typical reference tracking problem is achieved using FCS-MPC in this work (see Sec. IV-C). Rigorously, it can not be described using a transfer function since FCS-MPC is a nonlinear control law. A describing function (DF) method has been used to characterize the dynamics of FCS-MPC controlled power converters in [21]. The study implies that the dynamics of the FCS-MPC based inner control loop are much faster than the outer loop [21], [22], the control of power converters can be regarded as an ideal controlled voltage source to simplify the analysis of the higher tier control.

To understand the proposed control approach, the average global voltage $\bar{v}_{i}^{\text {bus }}$ is assumed to be obtained with the help of the bus voltage observer. $G_{c}(s)$ is able to eliminate the steadystate error since it has a pole approaches to zero. Hence, in the steady state, the input of the $G_{c}(s)$ is approximately zero, i.e $\Delta v_{\text {int }}=\left(v_{A e}-i_{i}^{o} \cdot R_{i}^{o}\right) \approx 0$. Discarding the residuals, we obtained,

$$
i_{i}^{o}=\frac{K_{v}\left(v_{\mathrm{dc}}^{*}-\bar{v}_{i}^{\mathrm{bus}}\right)}{R_{i}^{o}}
$$


Note $\bar{v}_{i}^{\text {bus }}=\bar{v}_{j}^{\text {bus }}$ establishes in finite time for all $i, j \in$ $\mathcal{V}$ as long as the consensus is achieved for all the agents. Consequently, the numerator of (11) is a constant if $K_{v}$ is identical for all the agents, i.e.

$$
i_{i}^{o} \cdot R_{i}^{o}=K_{v}\left(v_{\mathrm{dc}}^{*}-\bar{v}_{i}^{\text {bus }}\right)=\text { Constant. }
$$

Further, the load current can be shared proportionally to its power rating if

$$
\frac{R_{i}^{o}}{R_{j}^{o}}=\frac{S_{j}}{S_{i}}
$$

all $i, j \in \mathcal{V} . S_{i}$ and $S_{j}$ are the power ratings of the corresponding DG. (13) is a quite mild condition to be satisfied.

Moreover, an additional benefit of the proposed approach lies in its intrinsic voltage restoration capability. The following equation is obtained by some algebraic manipulation on (11)

$$
\bar{v}_{i}^{\text {bus }}=v_{\mathrm{dc}}^{*}-\frac{i_{i}^{o} \cdot R_{i}^{o}}{K_{v}} .
$$

From (14), one can conclude that the equivalent voltage droop is much reduced if $K_{v}$ is considerably large. Thus far, both voltage regulation and power-sharing are achieved simultaneously with a unified fashion. More specifically, only a voltage closed control loop with $i_{i}^{o} \cdot R_{i}^{o}$ as a feedback term is employed instead of applying two separate voltage and current regulators. This effectively simplifies the controller structure and tuning procedure, meanwhile theoretically cuts the communication bandwidth requirement in half.

Remark 1. As explained in Sec. I and II, the state-of-theart methods almost universally adopt two separate regulators. For a specific agent, it exchanges local bus voltage and output current information with its neighbors. Alternatively, the proposed approach only needs to exchange the estimated bus voltage information which reduces communication burden.

\section{B. Global Average Bus Voltage Observer}

To close the control loop, the global bus voltage needs to be estimated by each agent. As described in part C of Sec. III, the global bus voltage $\bar{v}_{i}^{\text {bus }}$ matches to the consensus state, while the local output voltage $v_{i}$ corresponds to the input signal of node $i$. Herein, the improved consensus observer of (7) is adopted for each individual agent. According to the notation of graph theory, the global dynamic consensus observer is

$$
\begin{aligned}
\overline{\boldsymbol{v}}^{\text {bus }} & =\boldsymbol{p}+\boldsymbol{v}, \\
\dot{\boldsymbol{p}} & =-\alpha \cdot \boldsymbol{p}-\boldsymbol{L}_{\mathrm{P}} \overline{\boldsymbol{v}}^{\text {bus }}+\boldsymbol{L}_{\mathrm{I}}^{\top} \boldsymbol{q}, \\
\dot{\boldsymbol{q}} & =-\boldsymbol{L}_{\mathrm{I}} \overline{\boldsymbol{v}}^{\text {bus }} .
\end{aligned}
$$

where $\boldsymbol{v}=\left[v_{1}, \ldots, v_{N}\right]^{\top}, \overline{\boldsymbol{v}}^{\text {bus }}=\left[\bar{v}_{1}^{\text {bus }}, \ldots, \bar{v}_{N}^{\text {bus }}\right]^{\top}, \boldsymbol{p}=$ $\left[p_{1}, \ldots, p_{N}\right]^{\top}, \boldsymbol{q}=\left[q_{1}, \ldots, q_{N}\right]^{\top}$.

This algorithm drives a group of agents to asymptotically converge to the average of the reference inputs. Note the presence of $\boldsymbol{L}_{\mathrm{I}}^{\top}$ does not bring more communication burden, since $\boldsymbol{L}_{\mathrm{I}}^{\top}=\boldsymbol{L}_{\mathrm{I}}$ for a undirected weight-balanced graph. In (15), the necessity of using explicit knowledge of the derivative of $\boldsymbol{v}$ is removed. More importantly, the special initialization requirement is eliminated, hence, enhancing the robustness of the algorithm.

\section{Full State-Variable FCS-MPC}

As the reference is generated by the outer loop, the remaining task is to track the reference through the manipulation of power converters. FCS-MPC has been proved a promising method for power converter control. It offers many advantages, e.g. being simple to apply in a multi-variable system and straightforward to include constraints. More importantly, superior dynamic performance can be obtained compared to the linear controller [23]-[26]. Therefore, FCS-MPC is selected in this work.

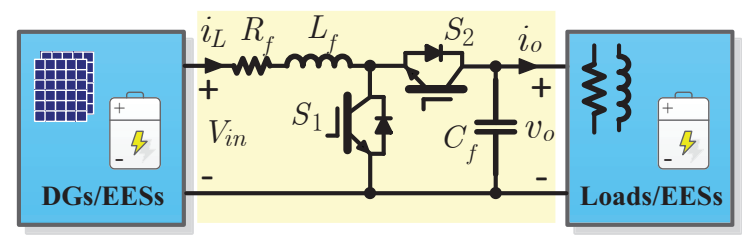

Fig. 5. Bidirectional Boost converter circuit.

Without losing generality, bidirectional Boost converters are considered as the interlinking converters (see Fig.5). As well investigated, the presence of right-half-plane (RHP) zero in the small-signal control-to-output transfer function makes it a formidable task to have a high control bandwidth. To overcome the sluggish dynamic response of the conventional control, a two-step full state-variable FCS-MPC algorithm is proposed in [27] and [28]. It is employed to achieve the reference tracking in this work. The recent work has proved that the fullstate variable FCS-MPC is able to overcome the drawback of non-minimum phase system, hence, to resolve the problem of voltage dip and poor dynamical performance of conventional control method during transient. For simplicity, the subscript $i$ for the $i$ th agent is omitted. The dynamics of the Boost converter is

$$
\begin{aligned}
& L_{f} \frac{\mathrm{d} i_{L}}{\mathrm{~d} t}=V_{i n}-R_{f} i_{L}-(1-u) v_{o}, \\
& C_{f} \frac{\mathrm{d} v_{o}}{\mathrm{~d} t}=(1-u) i_{L}-i_{o}
\end{aligned}
$$

where $V_{i n}$ is the input voltage. $L_{f}$ and $R_{f}$ correspond to the inductor and its equivalent serious resistor. $i_{L}$ represents the inductor current. $C_{f}$ is the output filter capacitor. $v_{o}$ and $i_{o}$ are the output voltage and output current, respectively. $u \in$ $\mathcal{U} \triangleq\{0,1\}$ denotes the input. $u=0$ if $S_{1}$ is turned off and $S_{2}$ is turned on, and $u=1$, otherwise. The discrete prediction equations can be obtained using forward Euler method

$$
\begin{aligned}
& i_{L}(k+1)=\left(1-\frac{R T_{s}}{L}\right) i_{L}(k)+\frac{T_{s}}{L}\left(V_{i n}(k)-(1-u(k)) v_{o}(k)\right), \\
& v_{o}(k+1)=v_{o}(k)+\frac{T_{s}}{C}\left((1-u(k)) \frac{i_{L}(k)+i_{L}(k+1)}{2}-i_{o}(k)\right) .
\end{aligned}
$$

For FCS-MPC, long-horizon might be desired for the applications with extremely low switching frequency to enhance system steady-state performance. Nevertheless, short-horizon usually achieves considerable satisfactory performance as the switching frequency reaches several $\mathrm{kHz}$. In this work, a two- 
step prediction horizon is considered. Then, the cost function is defined as

$$
\begin{aligned}
J= & \left\|\boldsymbol{x}(k+1)-\boldsymbol{x}^{*}(k+1)\right\|_{\boldsymbol{Q}}^{2} \\
& +\left\|\boldsymbol{x}(k+2)-\boldsymbol{x}^{*}(k+2)\right\|_{\boldsymbol{P}}^{2}+\sum_{\ell=k}^{k+1} \lambda_{u}\left\|\Delta u_{\ell}\right\|_{2}^{2},
\end{aligned}
$$

where $\boldsymbol{x}=\left[i_{L}, v_{o}\right]^{\top}$ are the state variables. $\boldsymbol{x}^{*}=\left[i_{L}^{*}, v^{*}\right]^{\top}$ are the reference of state variables. $\boldsymbol{Q}$ and $\boldsymbol{P}$ are the weighting matrix which are positive semi-definite. $\lambda_{u}>0$ is the weighting factor for switching effort, and $\Delta u_{\ell}=u_{\ell}-u_{\ell-1}$. $\|\xi\|_{\boldsymbol{Q}}^{2}=\xi^{\top} \boldsymbol{Q} \xi$ denotes the squared weighted Euclidean norm. To this end, the MPC optimal problem is formulated as

$$
\mathbf{U}(k)=\arg \min _{\mathbf{U}(k) \in \mathbb{U}} J,
$$

subject to: (17), and $u \in \mathcal{U} \triangleq\{0,1\}$,

where $\mathbf{U}(k)=[u(k), u(k+1)]^{\top} \in \mathcal{U} \times \mathcal{U}$.

For the optimal problem (19), the number of admissible control elements is $2^{2}=4$ in total. Therefore, the computational burden is mild, and the real-time implementation is practical. By enumerating all switching states, the optimal solution which minimizes the cost function (18) is obtained, and the first element is applied to the Boost converter. The full state-variable FCS-MPC algorithm can be depicted in the form of pseudo code in Algorithm 1, where $u_{o p}$ and $J_{o p}$ denote the optimal solution and optimal cost, respectively.

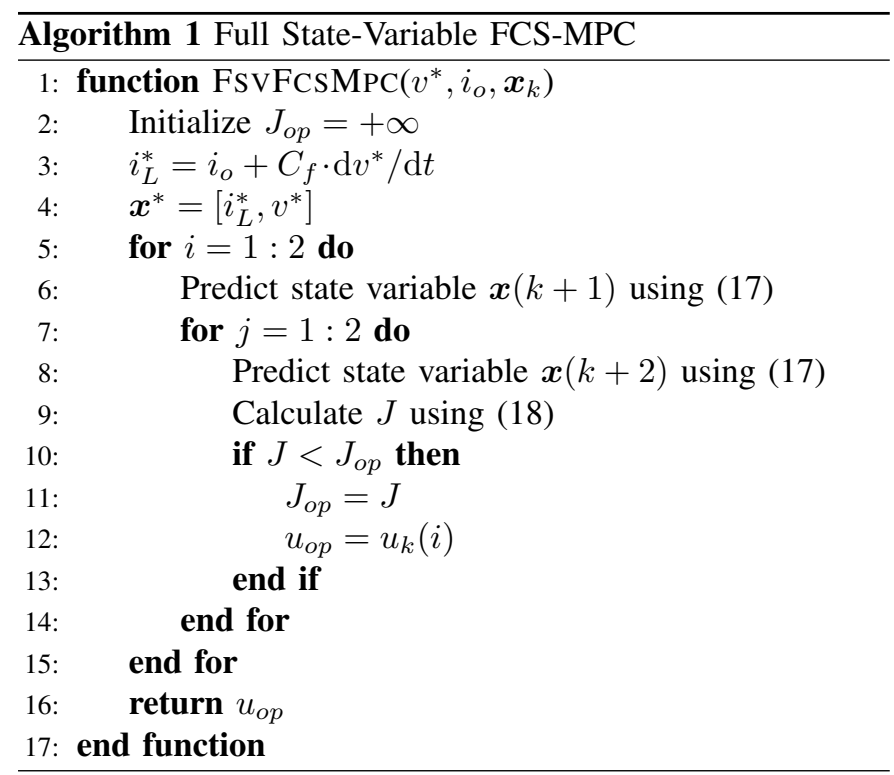

\section{Further Discussions}

Two aspects of interest in a dc microgrid are further discussed in this part. One is about the feasibility of the proposed algorithm with different power management strategy, such as optimal power dispatch. Recall (13), the internal virtual resistance is designed inversely proportional to their power ratings to realize power-sharing. Alternatively, it can be defined as a function of interested variable, typically, output power. Specifically, an optimal (economic) power dispatch problem can be defined as

$$
\min \sum_{i \in \mathcal{V}} C_{i}\left(P_{i}\right)
$$

The operating cost of the $i$-th DG are described by a quadratic function [14], [29], i.e.,

$$
C_{i}=a_{c i} P_{i}^{2}+b_{c i} P_{i}+c_{c i},
$$

where $a_{c i}, b_{c i}$, and $c_{c i}$ are the corresponding cost coefficients. Then the incremental cost is

$$
I C_{i}=2 a_{c i} P_{i}+b_{c i} .
$$

Without considering the power losses and power limit constraint, a unique optimal solution of (20) can be found as $I C_{i}=\lambda^{\star}$, where $\lambda^{\star}$ is the optimal incremental cost for DG units. Hence, the incremental cost of all DGs should be identical in the steady-state to achieve the optimal power dispatch. Motivated by the above observation, instead of using $i_{i}^{o} R_{i}^{o}$, let the incremental cost $I C_{i}$ as the feedback term. Then (12) becomes

$$
I C_{i}=K_{v}\left(v_{\mathrm{dc}}^{*}-\bar{v}_{i}^{\text {bus }}\right)=\text { Constant. }
$$

Therefore, all $I C_{i}$ converges to its optimal value by adjusting its output voltage, achieving the optimal power dispatch. The validation of the IC-based optimal power dispatch scheme is carried out in Sec. VI.

The other concern is about the output impedance characteristic. Usually, constant power load (CPL) is of interest in a dc microgrid since it shows a negative incremental resistance. This might introduce instability if not enough damping is provided. To date, virtual impedance is recognized as the most promising method to overcome this problem. Accordingly, it can be easily incorporated into the proposed control framework as shown in Fig. 6. In Sec. VI, the test results show the effectiveness of virtual impedance when CPLs are fed.

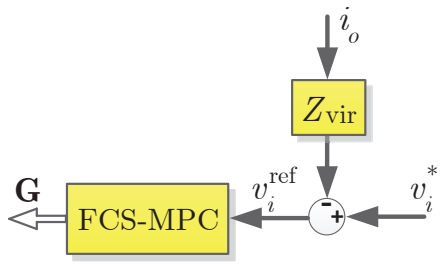

Fig. 6. Incorporating a virtual resistance into the proposed control framework, where $Z_{\text {vir }}$ denotes the virtual impedance.

Remark 2. The control variable $R_{i}^{o}$ proposed in this Sec. $I V$, namely internal virtual resistance, is intrinsically different from the concept of output virtual resistance $R_{\mathrm{vir}}$. In contrast to formulating the desired output impedance, the aim of accommodating $R_{i}^{o}$ is to guarantee power-sharing.

\section{Global Modeling and Stability Analysis}

Based on the proposed distributed controller, a global model of the DC microgrid is developed in this section. Then, the stability analysis is conducted. 


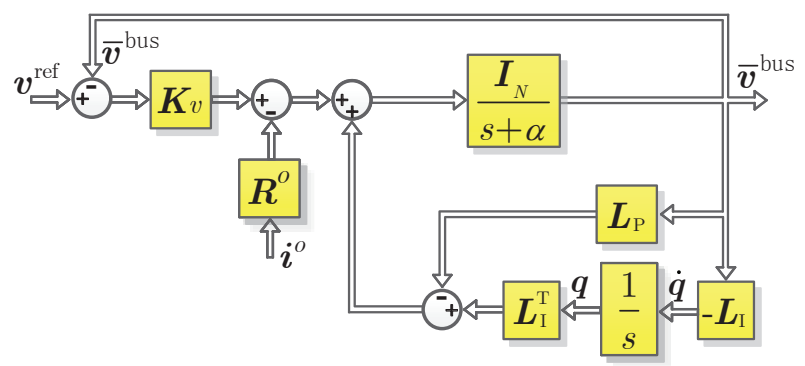

Fig. 7. The block diagram of the global DC microgrid model.

\section{A. Global Modeling}

Herein, the dynamics and non-linearity of the FCS-MPC controlled Boost converter are omitted, i.e, assuming that the output voltage of each converter tracks their reference without any error and delay. Then let $\boldsymbol{i}^{o}=\left[i_{1}^{o}, \ldots, i_{N}^{o}\right]^{\top}$ and $\boldsymbol{v}^{\text {ref }}=\left[v_{r e f}, \ldots, v_{r e f}\right]^{\top}$ be the global output current and global voltage reference. $\boldsymbol{R}^{o}=\operatorname{diag}\left\{R_{i}^{o}\right\}$ and $\boldsymbol{K}_{v}=\operatorname{diag}\left\{K_{v}\right\}$ are the feedback resistance matrix and the voltage error gain matrix, respectively.

The block diagram of the system global model is depicted in Fig. 7. Accordingly, the global dynamics can be obtained

$$
\dot{\boldsymbol{v}}=-\alpha \boldsymbol{v}+\boldsymbol{K}_{v}\left(\boldsymbol{v}^{\mathrm{ref}}-\overline{\boldsymbol{v}}^{\text {bus }}\right)-\boldsymbol{R}^{o} \boldsymbol{i}^{o} .
$$

Substitute (24) into global dynamic consensus equation (15), and define the new state variable $\boldsymbol{x}=\left[\overline{\boldsymbol{v}}^{\text {bus }}, \boldsymbol{q}, \boldsymbol{v}\right]^{\top}$. The global model of the DC microgrid can be obtained,

$$
\begin{aligned}
& \left(\begin{array}{c}
\dot{\boldsymbol{\boldsymbol { v }}}^{\text {bus }} \\
\dot{\boldsymbol{q}}
\end{array}\right)=\underbrace{\left(\begin{array}{cc}
-\alpha \boldsymbol{I}-\boldsymbol{L}_{\mathrm{P}}-\boldsymbol{K}_{v} & \boldsymbol{L}_{\mathrm{I}}^{\top} \\
-\boldsymbol{L}_{\mathrm{I}} & \mathbf{0}
\end{array}\right)}_{\boldsymbol{A}}\left(\begin{array}{c}
\overline{\boldsymbol{v}}^{\text {bus }} \\
\boldsymbol{q}
\end{array}\right) \\
& +\underbrace{\left(\begin{array}{c}
\boldsymbol{K}_{v} \\
\mathbf{0}
\end{array}\right)}_{\boldsymbol{B}} \boldsymbol{v}^{\mathrm{ref}}+\underbrace{\left(\begin{array}{c}
-\boldsymbol{R}^{o} \\
\mathbf{0}
\end{array}\right)}_{\boldsymbol{B}_{d}} \boldsymbol{i}^{o}=\boldsymbol{A} \boldsymbol{x}+\boldsymbol{B} \boldsymbol{u}+\boldsymbol{B}_{d} \boldsymbol{d}
\end{aligned}
$$

\section{B. Input-to-State Stability of DC Microgrids}

As observed, the associated global model of (25) is a Linear Time-Invariant (LTI) system. $\boldsymbol{v}^{\text {ref }}$ corresponds to the input $\boldsymbol{u}$, and $\boldsymbol{i}^{o}$ denotes the disturbance $\boldsymbol{d}$. Hence, the classical criterion of state-space stability can be employed.

Proposition: Input-to-State Stability (ISS) of (25). Let $\boldsymbol{L}_{\mathrm{P}}$ and $\boldsymbol{L}_{\mathrm{I}}$ be Laplacian matrices corresponding to strongly connected and weight-balanced undirected digraphs. Suppose that the observer parameter $\alpha>0$, and the input and disturbance are bound, i.e., $\sup _{0 \leq \tau \leq t}\left\|\boldsymbol{v}^{\mathrm{ref}}(\tau)\right\|<\infty$, and $\sup _{0 \leq \tau \leq t}\left\|\boldsymbol{i}^{\circ}(\tau)\right\|<\infty$. Then for any initial states $\boldsymbol{x}\left(t_{0}\right) \in$ $\mathbb{R}^{N}, \boldsymbol{q}\left(\bar{t}_{0}\right) \in \mathbb{R}^{N}$, the dynamics of (25) are ISS.

Proof: Let $\boldsymbol{r}=\mathbf{1} / \sqrt{n} \in \mathbb{R}^{N}$. Hence, $\boldsymbol{L}_{\mathrm{I}} \boldsymbol{r}=\boldsymbol{L}_{\mathrm{I}}^{\top} \boldsymbol{r}=\mathbf{0}$, where $\mathbf{1} \in \mathbb{R}^{N}$ denotes the vector of $n$ ones. Define $\boldsymbol{Q}_{r} \in$
$\mathbb{R}^{N \times(N-1)}$ be such that $\boldsymbol{T}_{r}=\left[\boldsymbol{r}, \boldsymbol{Q}_{r}\right]$ is an orthogonal matrix, i.e., $\boldsymbol{T}_{r} \boldsymbol{T}_{r}^{\top}=\boldsymbol{T}_{r}^{\top} \boldsymbol{T}_{r}=\boldsymbol{I}_{N}$. Consider the coordinate change

$$
\boldsymbol{z}=\boldsymbol{T}_{r}^{\top} \overline{\boldsymbol{v}}^{\text {bus }}, \quad \boldsymbol{y}=\boldsymbol{T}_{r}^{\top} \boldsymbol{q} .
$$

In the transformed coordinate, considering $\boldsymbol{r}^{\top} \boldsymbol{Q}_{r}=\mathbf{0}$ the dynamics of (25) become

$$
\begin{aligned}
\dot{\boldsymbol{z}} & =\left(\begin{array}{cc}
-\alpha-K_{v} & -\boldsymbol{r}^{\top} \boldsymbol{L}_{\mathrm{P}} \boldsymbol{Q}_{r} \\
\mathbf{0} & -\left(\alpha+K_{v}\right) \boldsymbol{I}_{N-1}-\boldsymbol{Q}_{r}^{\top} \boldsymbol{L}_{\mathrm{P}} \boldsymbol{Q}_{r}
\end{array}\right) \boldsymbol{z} \\
& +\left(\begin{array}{ccc}
\mathbf{0} & \mathbf{0} & \boldsymbol{0}^{\top} \\
\mathbf{0} & \boldsymbol{Q}_{r}^{\top} \boldsymbol{L}_{\mathrm{I}}^{\top} \boldsymbol{Q}_{r}
\end{array}\right) \boldsymbol{y}+\left(\begin{array}{l}
K_{v} \boldsymbol{r}^{\top} \\
K_{v} \boldsymbol{Q}^{\top}
\end{array}\right) \boldsymbol{v}^{\mathrm{ref}}-\left(\begin{array}{l}
R^{o} \boldsymbol{r}^{\top} \\
R^{o} \boldsymbol{Q}^{\top} \boldsymbol{i}^{o}
\end{array}\right), \\
\dot{\boldsymbol{y}} & =-\left(\begin{array}{cc}
0 & \mathbf{0} \\
\mathbf{0} & \boldsymbol{Q}_{r}^{\top} \boldsymbol{L}_{\mathrm{I}} \boldsymbol{Q}_{r}
\end{array}\right) \boldsymbol{z} .
\end{aligned}
$$

Note that the dynamic of the first element $y_{1}$ of $\boldsymbol{y}$ is $\dot{y}_{1}=0$, which means $y_{1}$ is a constant. This yields the conclusion that $y_{1}$ does not influence the stability of the system. By dropping $y_{1}$, one can obtain $\boldsymbol{y}_{2: N}=\left[y_{2}, \ldots, y_{N}\right]^{\top}=\boldsymbol{Q}_{r}^{\top} \boldsymbol{q}$. Then, the remaining system dynamics are

$$
\left(\begin{array}{c}
\dot{\boldsymbol{z}} \\
\dot{\boldsymbol{y}}_{2: N}
\end{array}\right)=\boldsymbol{A}_{\mathrm{Tr}}\left(\begin{array}{c}
\boldsymbol{z} \\
\boldsymbol{y}_{2: N}
\end{array}\right)+\boldsymbol{B}_{\mathrm{Tr}} \boldsymbol{v}^{\mathrm{ref}}+\boldsymbol{B}_{d, \operatorname{Tr}} \boldsymbol{i}^{o},
$$

where the corresponding matrices are shown at the bottom of this page. We have left to show that $\boldsymbol{A}_{\operatorname{Tr}}$ is Hurwitz.

One can observe that $\boldsymbol{A}_{\operatorname{Tr}}$ is block upper triangular. The upper left scalar block of $\left(-\alpha-K_{v}\right)$ is Hurwitz. The remaining $(2 n-2) \times(2 n-2)$ lower right block is written as

$$
\boldsymbol{F}=\left(\begin{array}{cc}
-\left(\alpha+K_{v}\right) \boldsymbol{I}_{N-1}-\boldsymbol{Q}_{r}^{\top} \boldsymbol{L}_{\mathrm{P}} \boldsymbol{Q}_{r} & \boldsymbol{Q}_{r}^{\top} \boldsymbol{L}_{\mathrm{I}} \boldsymbol{Q}_{r} \\
-\boldsymbol{Q}_{r}^{\top} \boldsymbol{L}_{\mathrm{I}} \boldsymbol{Q}_{r} & \mathbf{0}
\end{array}\right) .
$$

Invoking Lemma 1 and Lemma 2 (see appendix) [20], $\boldsymbol{F}$ is Hurwitz. Consequently, $\boldsymbol{A}_{\operatorname{Tr}}$ is Hurwitz, i.e., all eigenvalues of $\boldsymbol{A}_{\mathrm{Tr}}$ has strictly negative real part. The solution of (27) can be written as

$$
\begin{aligned}
\left(\begin{array}{c}
\boldsymbol{z}(t) \\
\boldsymbol{y}_{2: N}(t)
\end{array}\right) & =e^{\boldsymbol{A}_{\mathrm{Tr}} t}\left(\begin{array}{c}
\boldsymbol{z}(0) \\
\boldsymbol{y}_{2: N}(0)
\end{array}\right) \\
& +\int_{0}^{t} e^{\boldsymbol{A}_{\mathrm{Tr}}(t-\tau)}\left(\boldsymbol{B}_{\mathrm{Tr}} \boldsymbol{v}^{\mathrm{ref}}+\boldsymbol{B}_{d, \mathrm{Tr}} \boldsymbol{i}^{o}\right) d \tau
\end{aligned}
$$

For a Hurwitz matrix $\boldsymbol{A}_{\mathrm{Tr}}$, by using the bound

$$
\left\|e^{\boldsymbol{A}_{\mathrm{Tr}} t}\right\| \leq \kappa e^{-\underline{\lambda} t}, t \in \mathbb{R}_{\geq 0} .
$$

The determination of $\kappa$ and $\underline{\lambda}$ is given in [17] and [30], and can be set as

$$
\underline{\lambda}=-\lambda_{\max }\left(\boldsymbol{A}_{\operatorname{Tr}}\right), \kappa=1,
$$

$$
\boldsymbol{A}_{\mathrm{Tr}}=\left(\begin{array}{ccc}
-\alpha-K_{v} & -\boldsymbol{r}^{\top} \boldsymbol{L}_{\mathrm{P}} \boldsymbol{Q}_{r} & \mathbf{0} \\
\mathbf{0} & -\left(\alpha+K_{v}\right) \boldsymbol{I}_{N-1}-\boldsymbol{Q}_{r}^{\top} \boldsymbol{L}_{\mathrm{P}} \boldsymbol{Q}_{r} & \boldsymbol{Q}_{r}^{\top} \boldsymbol{L}_{\mathrm{I}} \boldsymbol{Q}_{r} \\
\mathbf{0} & -\boldsymbol{Q}_{r}^{\top} \boldsymbol{L}_{\mathrm{I}} \boldsymbol{Q}_{r} & \mathbf{0}
\end{array}\right), \boldsymbol{B}_{\mathrm{Tr}}=\left(\begin{array}{c}
K_{v} \boldsymbol{r}^{\top} \\
K_{v} \boldsymbol{Q}^{\top} \\
\mathbf{0}
\end{array}\right), \boldsymbol{B}_{d, \operatorname{Tr}}=\left(\begin{array}{c}
-R^{o} \boldsymbol{r}^{\top} \\
-R^{o} \boldsymbol{Q}^{\top} \\
\mathbf{0}
\end{array}\right)
$$


where $\lambda_{\max }\left(\boldsymbol{A}_{\mathrm{Tr}}\right)$ is the maximum eigenvalue of matrix $\boldsymbol{A}_{\mathrm{Tr}}$. An upper bound on the norm of the trajectories (30) for $t \in$ $\mathbb{R}_{\geq 0}$ is obtained as

$$
\begin{aligned}
& \left\|\left(\begin{array}{c}
\boldsymbol{z}(t) \\
\boldsymbol{y}_{2: N}(t)
\end{array}\right)\right\| \leq \kappa e^{-\underline{\lambda} t}\left\|\left(\begin{array}{c}
\boldsymbol{z}(t) \\
\boldsymbol{y}_{2: N}(t)
\end{array}\right)\right\| \\
& +\int_{0}^{t} \kappa e^{-\underline{\lambda}(t-\tau)}\left(\left\|\boldsymbol{B}_{\operatorname{Tr}}\right\|\left\|\boldsymbol{v}^{\mathrm{ref}}(\tau)\right\|+\left\|\boldsymbol{B}_{d, \operatorname{Tr}}\right\|\left\|\boldsymbol{v}^{\mathrm{ref}}(\tau)\right\|\right) \mathrm{d} \tau \\
& \leq \kappa e^{-\underline{\lambda} t}\left\|\left(\begin{array}{c}
\boldsymbol{z}(t) \\
\boldsymbol{y}_{2: N}(t)
\end{array}\right)\right\| \\
& +\frac{\kappa}{\underline{\lambda}}\left(\left\|\boldsymbol{B}_{\operatorname{Tr}}\right\| \sup _{0 \leq \tau \leq t}\left\|\boldsymbol{v}^{\mathrm{ref}}(\tau)\right\|+\left\|\boldsymbol{B}_{d, \operatorname{Tr}}\right\| \sup _{0 \leq \tau \leq t}\left\|\boldsymbol{i}^{o}(\tau)\right\|\right) .
\end{aligned}
$$

Since $\sup _{0 \leq \tau \leq t}\left\|\boldsymbol{v}^{\text {ref }}(\tau)\right\|<\infty$ and $\sup _{0 \leq \tau \leq t}\left\|\boldsymbol{i}^{\mathrm{o}}(\tau)\right\|<\infty$, the zero-input response decays to zero exponentially, whereas the zero-state response is bounded for every bounded input and disturbance. Therefore, the dynamics of (25) are ISS.

\section{IMPLEMENTATION AND VALIDATION}

Real-time HiL test of a low voltage autonomous DC microgrid is used to validate the proposed control strategy. The test is implemented on PLECS RT Boxes (see Fig. 8). One of the RT Box is used to emulates the power stage of the dc microgrid. The switching behavior of the power converters is modeled to obtain accurate results. The other one is used as a real-time controller to run the proposed cooperative control algorithm. The PWM signals generated by the controller are captured by the plant emulator with a time resolution of less than $10 \mathrm{~ns}$. Then, the controller receives the feedback signals coming from the plant emulator using analog-to-digital (AD) input channels. Therefore, the test bench is close to the physical system considering the time delays of sampling, transmission, and calculation.

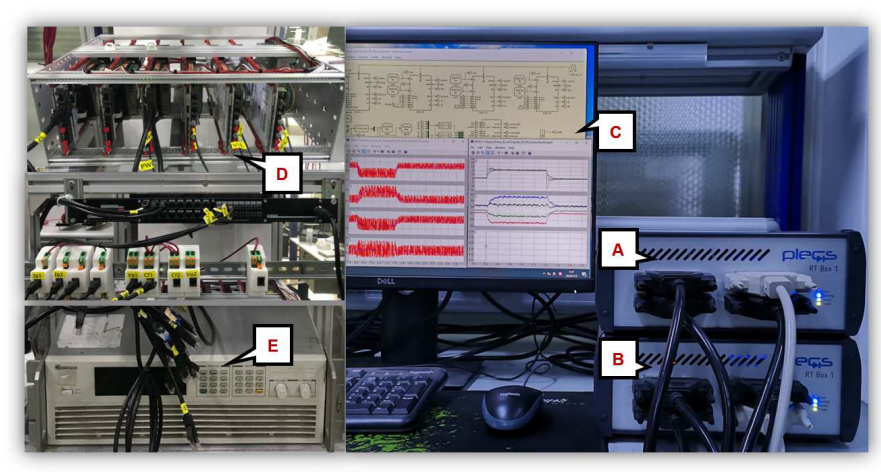

Fig. 8. Real-time HiL and experimental test bench. A: Plant emulator. B: Realtime controller. C: Monitor. D: Power conversion system. (power converters, sensors, and interfaces, which are not used in this work.) E: Power supply.

The system consisting of four DG units with its corresponding communication network (see Fig.9). The feeder impedances are modeled by series-connected inductance and resistance. Then common loads are fed by all DGs via interfacing DC-DC converters. The local load-1 to load-4 are $20 \Omega, 30 \Omega, 30 \Omega$ and $40 \Omega$, respectively. The common bus load is initially $20 \Omega$. Other key parameters and the controller coefficients are given in TABLE II.

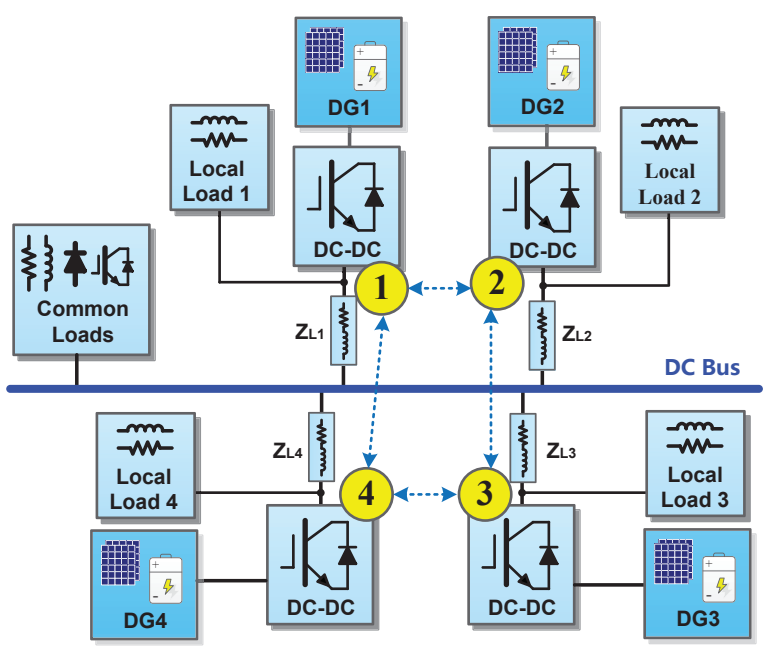

Fig. 9. The set-up of a low voltage DC microgrid test bench and its corresponding communication network topology.

TABLE II

KEY PARAMETERS OF THE TEST BENCH

\begin{tabular}{l|c}
\hline \hline Parameters & Value \\
\hline Bus Nominal Voltage: $V_{r e f}$ & $88 \mathrm{~V}$ \\
\hline Power Rating Ratio & $1: 1: 1: 1$ \\
\hline Feeder Impedance: $Z_{L 1}$ & $R=0.1 \Omega, L=100 \mu H$ \\
\hline Feeder Impedance: $Z_{L 2}$ & $R=0.8 \Omega, L=200 \mu H$ \\
\hline Feeder Impedance: $Z_{L 3}$ & $R=0.25 \Omega, L=150 \mu H$ \\
\hline Feeder Impedance: $Z_{L 4}$ & $R=0.5 \Omega, L=80 \mu H$ \\
\hline \hline Proportional Gain Matrix: $\boldsymbol{K}_{v}$ & $\operatorname{diag}\{200\}$ \\
\hline Output Resistance Matrix: $\boldsymbol{R}^{o}$ & $\operatorname{diag}\{10\}$ \\
\hline Incremental cost coefficients: $a_{c 1 \sim 4}$ & $0.05,0.025,0.03,0.01$ \\
\hline Incremental cost coefficients: $b_{c 1 \sim 4}$ & $5,10,10,20$ \\
\hline Data Exchange Period: $T_{\text {com }}$ & $4 \mathrm{~ms}$ \\
\hline
\end{tabular}

\section{A. Implementation}

Before evaluating the controller performance, several critical implementation issues from an engineering perspective are considered and summarized as following design guidelines.

Guideline 1. The output voltage and the internal state variable (i.e. inductor current) of the power converter should be strictly limited below the predefined protection threshold for safety consideration. This can be easily realized by adding soft constraints to the cost function of FCS-MPC controller [22].

Guideline 2. The consensus algorithm is implemented in a low refresh rate due to communication bandwidth restriction. Whereas local measurement of the output voltage $v_{o}$ has a much higher updating rate. Feeding $v_{o}$ into a lower pass filter is an effective solution for removing switching noise, and therefore, enhancing system performance.

Guideline 3. The compensator of $G_{c}(s)$ shown in (9) should be reset at the instant of enabling the corresponding converter, to eliminate the non-zero initial disturbance.

\section{B. Comparative study}

Fig. 10 comparatively evaluates the control performance of the scheme proposed in [13] and the proposal in this work. The 


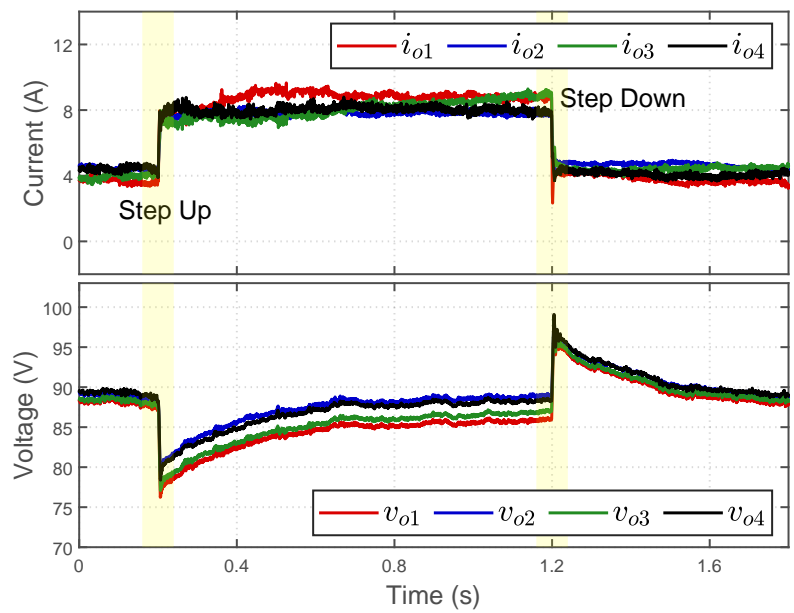

(a) Performance of a distributed cooperative control proposed in [13].

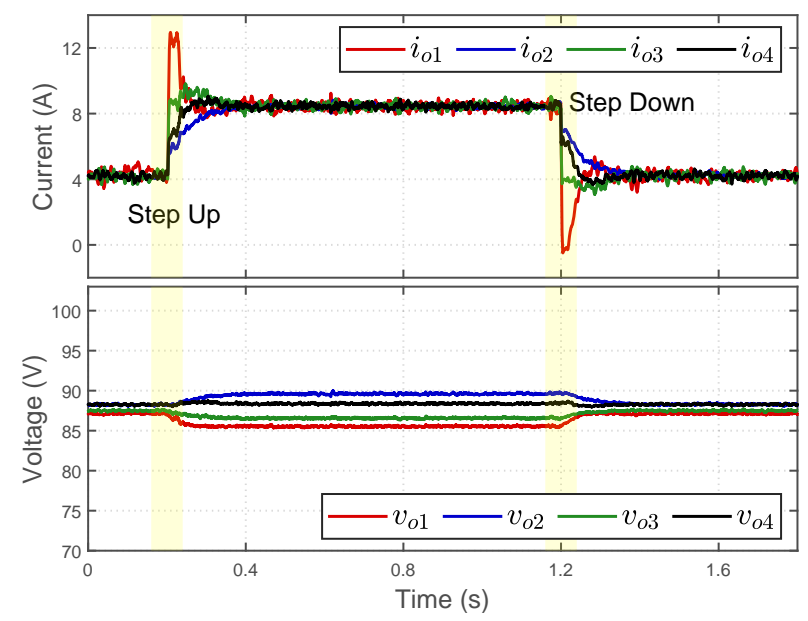

(b) Performance of the proposed control strategy in this work.

Fig. 10. Comparative performance evaluation.

test condition is the same for both methods. A load step change disturbance is conducted to investigate the voltage regulation and power-sharing performance during transient process. At $t=0.2 \mathrm{~s}$, a step load change on the common bus from 20 $\Omega$ to $4 \Omega$ happens, and then steps back at $t=1.2 s$. From top to bottom are output currents and output voltages of each DG. As observed, both methods achieve voltage regulation and power-sharing in the steady-state. Nevertheless, the control scheme proposed in [13] has a sudden voltage drop and rise during the load changes. A relatively long regulation process is observed which degrades the power quality. In contrast, the proposed control strategy can achieve the new current sharing status rapidly without drastic voltage changes. In general, the proposed solution outperforms its counterpart in terms of voltage regulation during load changes.

\section{Resiliency of Communication Failure}

A communication failure between DG-1 and DG-2 is deliberately configured and tested (see Fig. 9). To minimize the effect of communication failure, we design a time-varying communication graph by employing a handshake mechanism. In particular, a handshake flag is transmitted with the shared

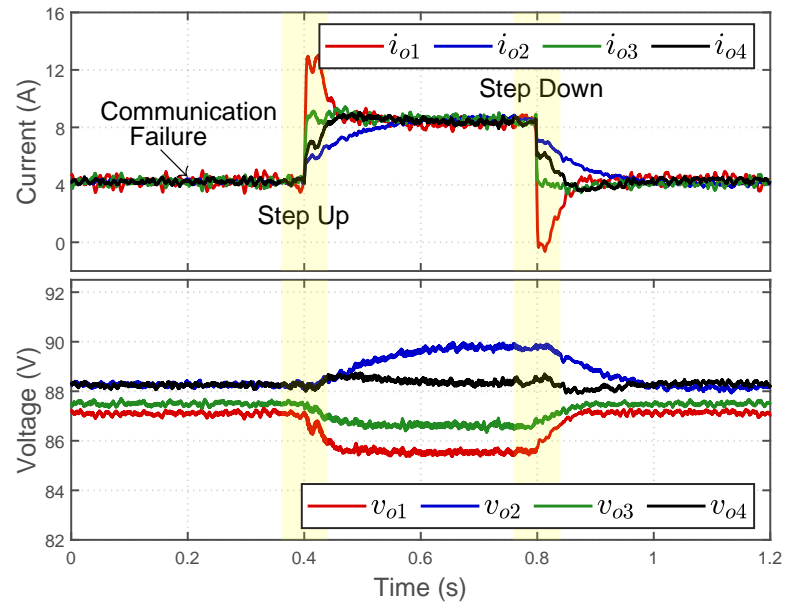

Fig. 11. Communication failure resiliency evaluation.

information between each neighbor agents. The departure of the flag indicates a communication failure between the agents. Then, the corresponding elements of the adjacent matrix $\boldsymbol{A}_{G}$ is set as 0 . Meanwhile, the remaining connection is changed accordingly to maintain a constant in-degree matrix $D^{\text {in }}$. This method entails a minimum influence on the observer during the transient process of the communication topology. For instance, the communication between DG-1 and DG-2 is lost at $t=0.2 s$. Then, in agent- $1, a_{21}$ becomes 0 , while $a_{41}$ times 2 to maintain $d_{i}^{\text {in }}$ unchanged. Afterward, the load step-up and step-down happens at $0.4 s$ and $0.8 s$, respectively.

The test result is interpreted in Fig. 11. Thanks to the optimal redundancy design of the cyber network, the link failure will not break the graphical connectivity. The consensus of the average voltage across the microgrid is still shared by all DGs. Besides, the designed handshake mechanism guarantees a smooth transition. As depicted in Fig. 11, the DGs achieve voltage regulation and power-sharing during the load change transient processes. Consequently, the performance is not compromised under such a fault condition. This test result indicates that the proposed control scheme is resilient to communication failure.

\section{Evaluation of Plug-and-Play Capability}

The plug-and-play capability is evaluated by disabling and enabling both communication and output power of a DG unit. The same handshake mechanism is employed. Specifically, the power converter of DG-1 shuts down at $t=0.2 s$ immediately due to a failure of the power converter. It is plugged-in at $t=0.6 \mathrm{~s}$ assuming the fault is removed. Fig. 12 shows the dynamic process. As one can observe, the remaining converters automatically re-distribute the load accurately when DG-1 shuts down. The remaining average bus voltage is regulated around the rated value. However, agent- 1 is excluded from group, and the voltage on bus- 1 is determined by its external circuit. Accordingly, as the DG-1 plugs back, the communication is established again. Afterward, all four DGs share the load automatically without compromising voltage regulation performance. 


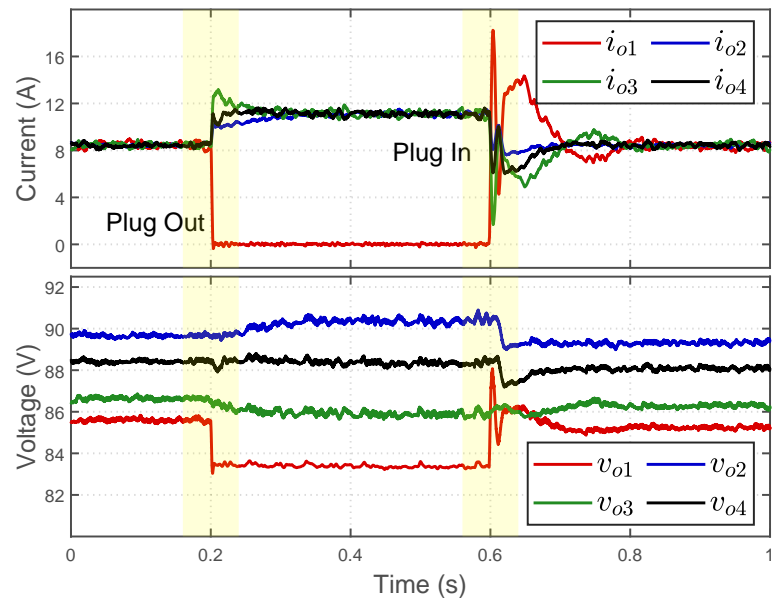

Fig. 12. Plug-and-play capability evaluation of the proposed controller.

\section{E. Effect of Message Update Rate}

The realistic implementation of the dynamic consensus algorithm is usually realized using the Euler discretization method. The information update rate can be altered according to the communication configuration. Therefore, it makes considerable engineering sense to evaluate the control performance under different message update rate. Another two scenarios of data exchange periods are evaluated. The test results with $T_{\text {com }}=1 \mathrm{~ms}$ and $T_{\text {com }}=8 \mathrm{~ms}$ are shown in Fig. 13. From the observation of the results, the output currents converge to the identical value under both situations. With the increase of $T_{\text {com }}$, the steady-state current ripple increases, meanwhile the settling time becomes longer. Nevertheless, as the message update rate $T_{\text {com }}$ increases to $8 \mathrm{~ms}$, the controller still achieves acceptable load sharing performance.

\section{F. Load Change on a local bus}

The feeder impedance affects the power-sharing performance directly. To examine the control performance with respect to the variation of feeder impedance, an extreme test condition, i.e., load change occurs on the local bus-2, is carried out. The result is shown in Fig. 14. The output current of DG-2 has the greatest surge since the transient power is directly provided by this node. Nevertheless, the proposed control strategy is independent of microgrid parameters. The unbalanced output currents are eliminated rapidly, meanwhile, the average voltage is maintained around the rated value.

\section{G. Evaluation of optimal power dispatch}

As discussed, the proposed control strategy is able to accommodate other power management flexibly. Two typical scenarios are tested. The first one assumes the power rating ratio of the DGs is $1: 1: 2: 2$. Then, the proportional powersharing can be achieved by setting $R_{1}^{o}: R_{2}^{o}: R_{3}^{o}: R_{4}^{o}=$ $2: 2: 1: 1$. Fig. 15(a) shows that the control objectives are accomplished considering the load disturbance.

The second test is to validate the IC-based optimal power dispatch (see Sec. IV-D). The incremental cost based optimal power dispatch is test by applying the IC as the feedback term.

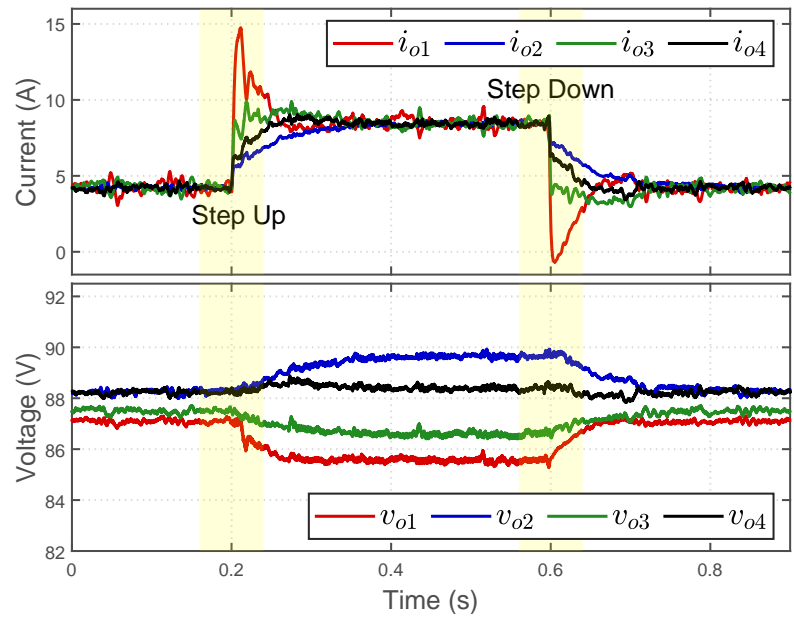

(a) $T_{\mathrm{com}}=1 \mathrm{~ms}$

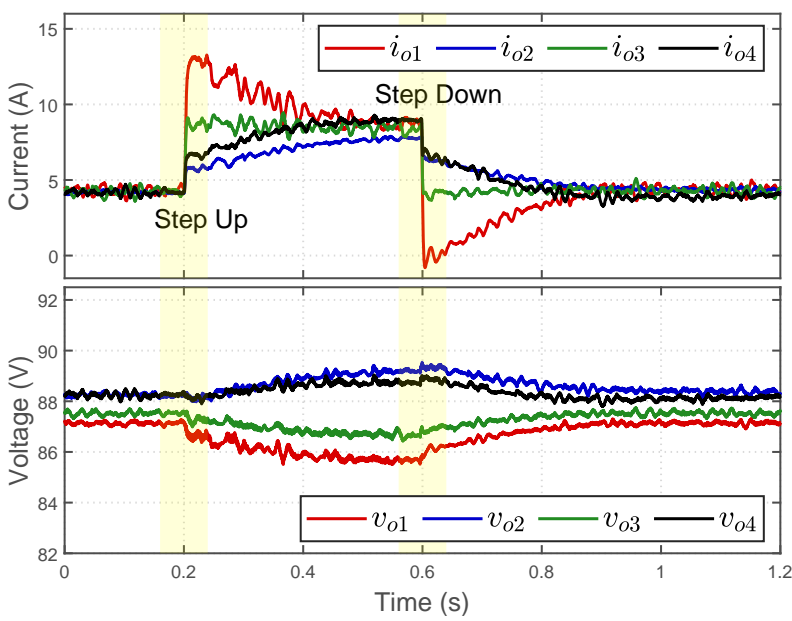

(b) $T_{\text {com }}=8 \mathrm{~ms}$

Fig. 13. Effect of message update rate evaluation.

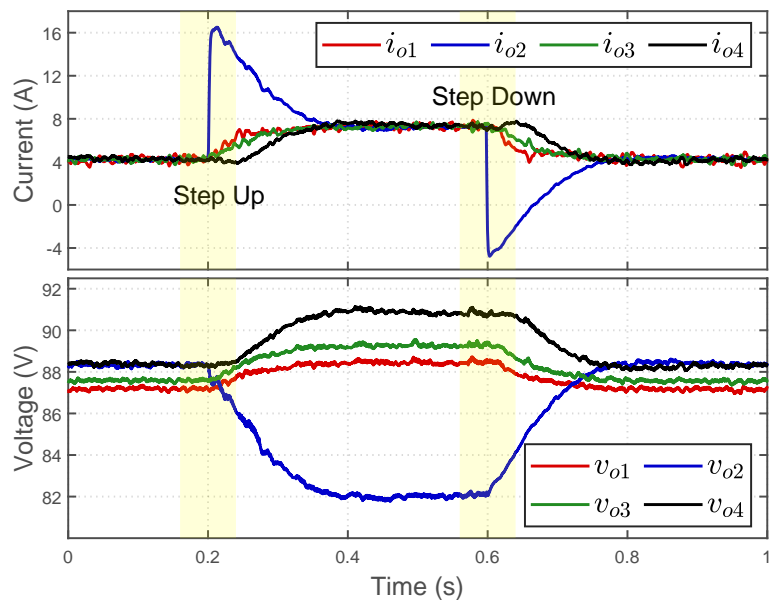

Fig. 14. Evaluation of load change on a local bus.

Fig. 15(b) illustrates the DG's output power, output voltage, and incremental cost. The incremental cost coefficients are collected in TABLEII. As expected, the ICs are converge to the optimal solution rapidly governed by the cooperative controller, realizing the optimal power dispatch. 


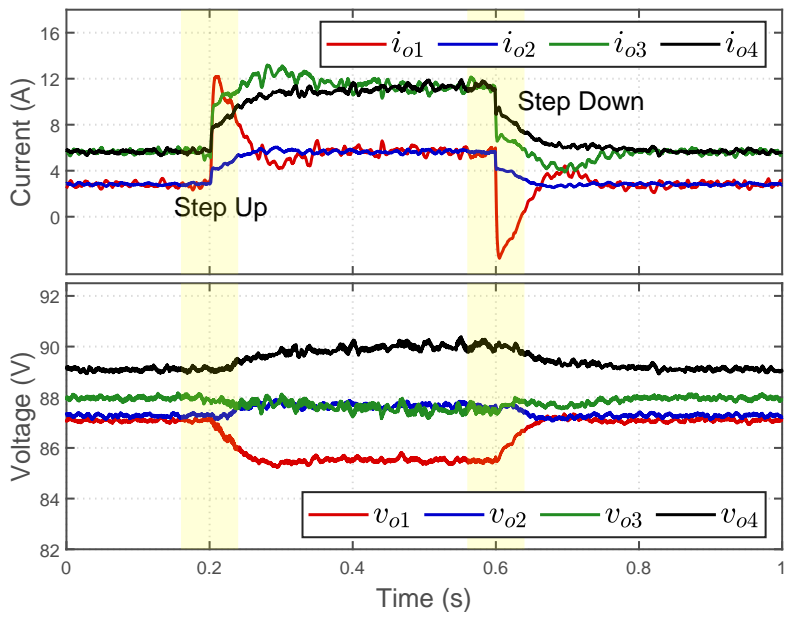

(a) Different power ratings.

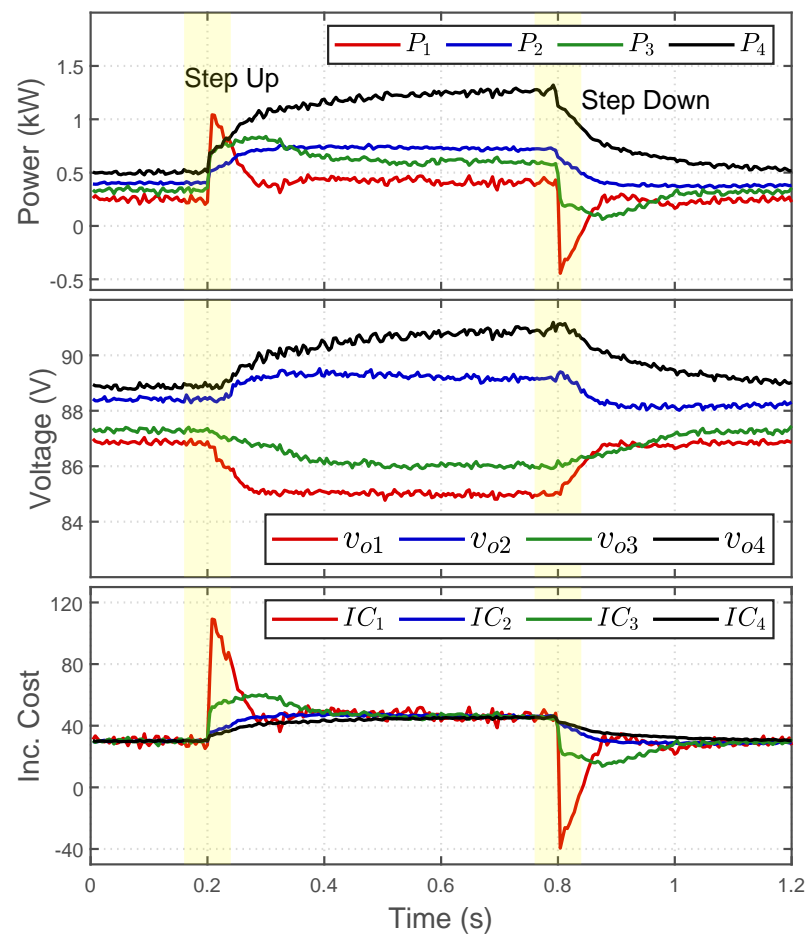

(b) Incremental cost based optimal power dispatch

Fig. 15. Evaluation of optimal power dispatch.

\section{H. Evaluation of feeding constant power loads}

As discussed in Sec. IV-D, the output virtual impedance can be integrated into the proposed framework. To test its effectiveness, a CPL connected to the common bus is considered. Since the main contribution of this work is to propose a distributed cooperative controller for a dc microgrid, a simple virtual resistor (VR) is designed for this test. The resistance of the feeder impedance is reduced to $0.05 \Omega$, where the damping is not sufficient. Fig. 16 depicts the test results. VR is disabled at the beginning of the test, and insufficient damping is provided in this situation, leading to a highly distorted output current. To damp the system, a $0.25 \Omega$ VR is inserted at $t=0.6 \mathrm{~s}$. As expected, the output current of each DG converges to the average value, and the oscillations are mitigated effectively. A

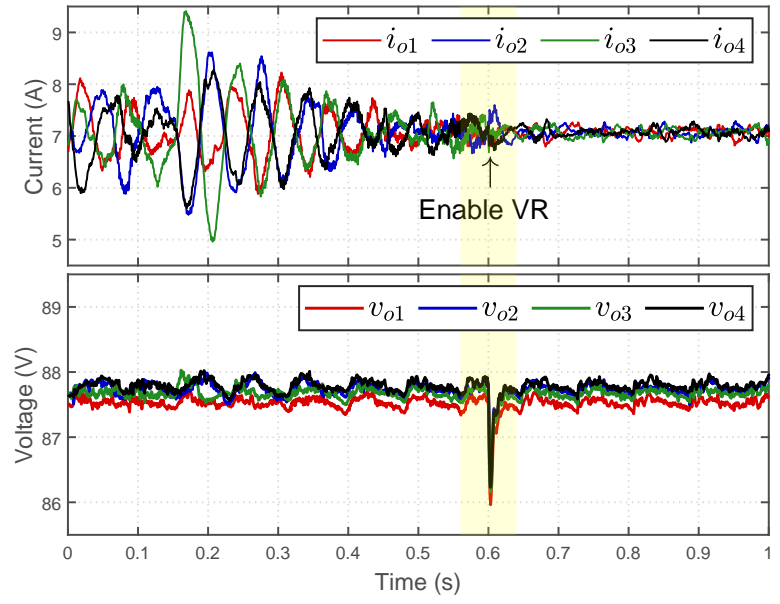

Fig. 16. Evaluation of feeding CPLs.

sudden voltage drop is observed at the instant of activating the VR. Nevertheless, the output voltages are restored rapidly due to the voltage regulation capability of the proposed cooperative controller. This shows the effectiveness of the VR.

\section{Evaluation of over current protection function}

As discussed in the implementation of Guideline 1, the internal inductor current should be limited to a predefined level to guarantee the safe operation of the power converter. This can be realized by adding soft constraints to the cost function of FCS-MPC. To validate this function, a $12 \mathrm{~A}$ inductor current threshold is configured. Two test scenarios, i.e., (a) hard protection without current limiter and (b) soft protection with current limiter, are conducted. The test results are presented in Fig. 17. As one can observe, when the current limiter function is disabled, DG-2 is shunted down as the inductor current exceeds the threshold during the process of load change at $t=0.2 s$. DG-2 is not able to return to the normal operation until a restart command is received. On the contrary, the inductor current can be limited below its threshold strictly, i.e. $12 \mathrm{~A}$, instead of shunting down the power converter. This soft protection mechanism allows DG-2 to offer the most power it can provide while assuring its safe operation. Therefore, this function enhances the resiliency of the system.

\section{CONCLUSION}

In this work, a distributed cooperative control approach is proposed for islanded DC microgrids. In particular, an improved dynamic consensus algorithm is employed to estimate the average global bus voltage at each distributed agent. Both voltage regulation and load sharing control objectives are achieved using a unified voltage closed-loop controller. Thereby, the proposed approach simplifies the controller structure and facilitates the parameter design. Thereafter, we use a full state-variable MPC by manipulating the power switches directly to achieve rapid reference tracking. Moreover, the global model of the DC microgrid considering the cyber network is established. Based on this, the input-to-state stability analysis is conducted. To the end, implementation guidelines are given, and comprehensive real-time HiL test is illustrated 


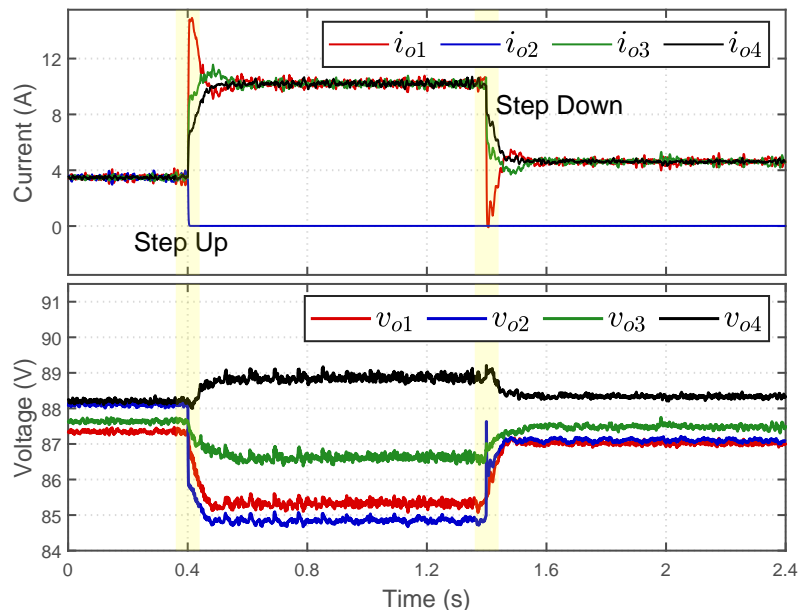

(a) Protection function test without internal inductor current limiter.

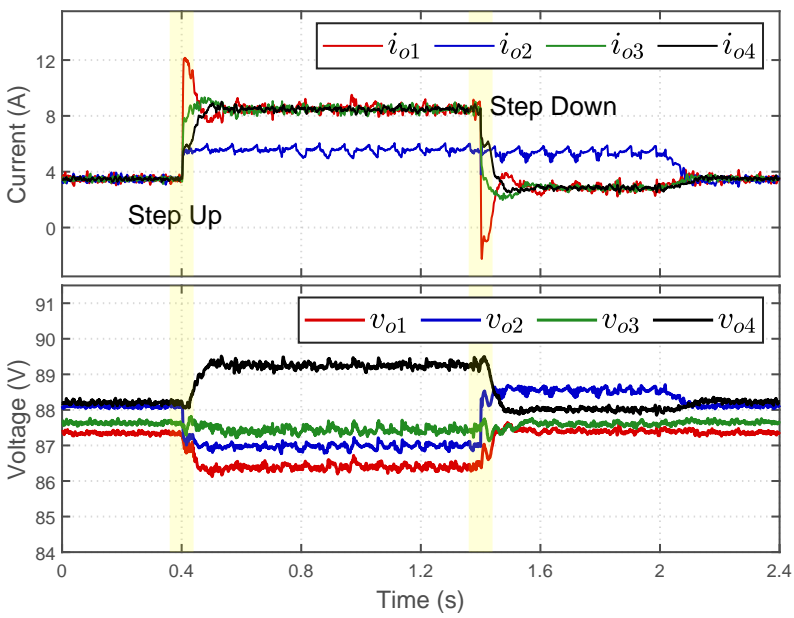

(b) Protection function test with internal inductor current limiter.

Fig. 17. Comparative study of over current protection function.

for different operating conditions. The proposed approach exhibits superior performance, considering the mitigation of voltage deviation, the accurate load sharing, the capacity of plug-and-play, the robustness to message update rate, and the resiliency to communication failure.

\section{APPENDIX}

The following two lemmas are found in [20]. To facilitate readability, they are re-written here.

Lemma 1: Suppose matrix $\boldsymbol{A}, \boldsymbol{B} \in \mathbb{R}^{p \times p}$ are such that $\boldsymbol{A}+\boldsymbol{A}^{\top}<0$ and $\boldsymbol{B}$ is invertible. Then the matrix

$$
M=\left(\begin{array}{cc}
A & B^{\top} \\
-B & 0
\end{array}\right)
$$

\section{is Hurwitz.}

Lemma 2: Suppose the matrix $\boldsymbol{A} \in \mathbb{R}^{p \times p}$ has rank $p-1$, and let $\ell, r \in \mathbb{R}^{p}$ be left and right eigen-vectors, respectively of its zero eigenvalue. Let $\boldsymbol{B} \in \mathbb{R}^{p \times(p-1)}$ be a marix whose columns form a basis for span $\{\ell\}^{\perp}$ (be orthogonal with $\ell$ ), and let $C \in \mathbb{R}^{p \times(p-1)}$ whose columns form a basis for span $\{r\}^{\perp}$ (be orthogonal with $r$ ). Then $\boldsymbol{B}^{\top} \boldsymbol{A} \boldsymbol{C}$ is invertible.

\section{REFERENCES}

[1] Q.-C. Zhong, "Virtual synchronous machines: A unified interface for grid integration," IEEE Power Electron. Mag., vol. 3, no. 4, pp. 18-27, Dec. 2016.

[2] J. M. Guerrero, J. C. Vasquez, J. Matas, L. G. De Vicuña, and M. Castilla, "Hierarchical control of droop-controlled ac and dc microgrids-a general approach toward standardization," IEEE Trans. Ind. Electron., vol. 58, no. 1, pp. 158-172, Jan. 2011.

[3] G. Lou, W. Gu, Y. Xu, M. Cheng, and W. Liu, "Distributed mpc-based secondary voltage control scheme for autonomous droop-controlled microgrids," IEEE Trans. Sustain. Energy, vol. 8, no. 2, pp. 792-804, Apr. 2017

[4] F. Dörfler, J. W. Simpson-Porco, and F. Bullo, "Breaking the hierarchy: Distributed control and economic optimality in microgrids," IEEE Control Netw. Syst., vol. 3, no. 3, pp. 241-253, Sep. 2016.

[5] Q. Xu, X. Hu, P. Wang, J. Xiao, P. Tu, C. Wen, and M. Y. Lee, "A decentralized dynamic power sharing strategy for hybrid energy storage system in autonomous dc microgrid," IEEE Trans. Ind. Electron., vol. 64 no. 7, pp. 5930-5941, 2017.

[6] Y. Xia, W. Wei, M. Yu, X. Wang, and Y. Peng, "Power management for a hybrid ac/dc microgrid with multiple subgrids," IEEE Trans. Power Electron., vol. 33, no. 4, pp. 3520-3533, Apr. 2018.

[7] H. T. V. and L. Hong-Hee, "Accurate power sharing with harmonic power for islanded multibus microgrids," IEEE Trans. Emerg. Sel. Topics Power Electron., vol. 7, no. 2, pp. 1286-1299, Jun. 2019.

[8] F. Guo, L. Wang, C. Wen, D. Zhang, and Q. Xu, "Distributed voltage restoration and current sharing control in islanded dc microgrid systems without continuous communication," IEEE Trans. Ind. Electron., vol. 67, no. 4, pp. 3043-3053, Apr. 2020.

[9] J. W. Simpson-Porco, Q. Shafiee, F. Dörfler, J. C. Vasquez, J. M. Guerrero, and F. Bullo, "Secondary frequency and voltage control of islanded microgrids via distributed averaging," IEEE Trans. Ind. Electron., vol. 62, no. 11, pp. 7025-7038, Nov. 2015.

[10] X. Lu, J. M. Guerrero, K. Sun, and J. C. Vasquez, "An improved droop control method for dc microgrids based on low bandwidth communication with dc bus voltage restoration and enhanced current sharing accuracy," IEEE Trans. Power Electron., vol. 29, no. 4, pp. 1800-1812, Apr. 2014.

[11] P. Wang, X. Lu, X. Yang, W. Wang, and D. Xu, "An improved distributed secondary control method for dc microgrids with enhanced dynamic current sharing performance," IEEE Trans. Power Electron., vol. 31, no. 9, pp. 6658-6673, Sep. 2016.

[12] J. Engels, H. Almasalma, and G. Deconinck, "A distributed gossip-based voltage control algorithm for peer-to-peer microgrids," in 2016 IEEE International Conference on Smart Grid Communications (SmartGridComm). IEEE, 2016, pp. 370-375.

[13] V. Nasirian, S. Moayedi, A. Davoudi, and F. L. Lewis, "Distributed cooperative control of dc microgrids," IEEE Trans. Power Electron., vol. 30, no. 4, pp. 2288-2303, Apr. 2015.

[14] A. A. Hamad, M. A. Azzouz, and E. F. El-Saadany, "Multiagent supervisory control for power management in dc microgrids," IEEE Trans. Smart Grid, vol. 7, no. 2, pp. 1057-1068, Mar. 2016.

[15] B. Fan, S. Guo, J. Peng, Q. Yang, W. Liu, and L. Liu, "A consensusbased algorithm for power sharing and voltage regulation in dc microgrids," IEEE Trans. Ind. Informat., vol. 16, no. 6, pp. 3987-3996, Jun. 2020.

[16] S. Trip, M. Cucuzzella, X. Cheng, and J. Scherpen, "Distributed averaging control for voltage regulation and current sharing in dc microgrids," IIEEE Contr. Syst. Lett., vol. 3, no. 1, pp. 174-179, Jan. 2019.

[17] S. S. Kia, B. Van Scoy, J. Cortes, R. A. Freeman, K. M. Lynch, and S. Martinez, "Tutorial on dynamic average consensus: The problem, its applications, and the algorithms," IEEE Control Syst. Mag., vol. 39, no. 3, pp. 40-72, Jun. 2019.

[18] D. P. Spanos, R. Olfati-Saber, and R. M. Murray, "Dynamic consensus on mobile networks," in IFAC world congress. Citeseer, 2005, pp. 1-6.

[19] M. Cucuzzella, S. Trip, C. De Persis, X. Cheng, A. Ferrara, and A. van der Schaft, "A robust consensus algorithm for current sharing and voltage regulation in de microgrids," IEEE Trans. Control Syst. Technol., vol. 27, no. 4, pp. 1583-1595, Jul. 2019.

[20] R. A. Freeman, P. Yang, and K. M. Lynch, "Stability and convergence properties of dynamic average consensus estimators," in Proceedings of the 45th IEEE Conference on Decision and Control. IEEE, 2006, pp. 338-343.

[21] T. Dragičević, C. Zheng, J. Rodriguez, and F. Blaabjerg, "Robust quasipredictive control of $l c l$-filtered grid converters," IEEE Trans. Power Electron., vol. 35, no. 2, pp. 1934-1946, Feb. 2020. 
[22] T. Dragičević, "Model predictive control of power converters for robust and fast operation of ac microgrids," IEEE Trans. Power Electron., vol. 33, no. 7, pp. 6304-6317, Jul. 2018.

[23] J. Rodriguez, M. P. Kazmierkowski, J. R. Espinoza, P. Zanchetta H. Abu-Rub, H. A. Young, and C. A. Rojas, "State of the art of finite control set model predictive control in power electronics," IEEE Trans. Ind. Informat., vol. 9, no. 2, pp. 1003-1016, May 2013.

[24] P. Karamanakos and T. Geyer, "Guidelines for the design of finite control set model predictive controllers," IEEE Trans. Power Electron., Jul. 2020.

[25] Z. Zhang, H. Fang, F. Gao, J. Rodríguez, and R. Kennel, "Multiplevector model predictive power control for grid-tied wind turbine system with enhanced steady-state control performance," IEEE Trans. Ind. Electron., vol. 64, no. 8, pp. 6287-6298, Aug. 2017.

[26] Z. Zhang, Z. Li, M. P. Kazmierkowski, J. Rodríguez, and R. Kennel, "Robust predictive control of three-level npc back-to-back power converter pmsg wind turbine systems with revised predictions," IEEE Trans. Power Electron., vol. 33, no. 11, pp. 9588-9598, Nov. 2018.

[27] Y. Li, F. Grimm, and Z. Zhang, "Model predictive control for three-phase four-wire voltage source converters in standalone mode," in $201921 \mathrm{st}$ European Conference on Power Electronics and Applications (EPE'19 ECCE Europe). IEEE, 2019, pp. P.1-P.8.

[28] Y. Li, Z. Zhang, and R. Kennel, "A full state-variable predictive control of bi-directional boost converters with guaranteed stability," in $202022 s t$ European Conference on Power Electronics and Applications (EPE'20 ECCE Europe). IEEE, 2020, pp. -.

[29] P. Lin, C. Jin, J. Xiao, X. Li, D. Shi, Y. Tang, and P. Wang, "A distributed control architecture for global system economic operation in autonomous hybrid ac/dc microgrids," IEEE Trans. Smart Grid, vol. 10, no. 3, pp. 2603-2617, May 2019

[30] D. S. Bernstein, Matrix mathematics: theory, facts, and formulas. Princeton university press, 2009.

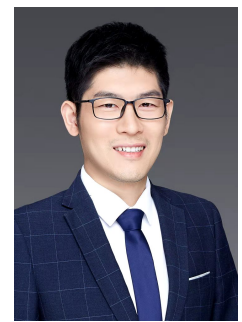

Yu Li (Student Member, IEEE), was born in Shandong, China. He received the M.S. degree from the School of Automation, Northwestern Polytechnical University, Xi'an, China, in 2012. From 2012 to 2013, he has been working as a servo drive engineer at Bosch Rexroth, Xian, China. From 2013 to 2015, he was a research engineer in the Department of Electrical and Computer Engineering, Kettering University, Flint, MI, USA. From 2016 to 2018, he joined State Grid Intelligence Technology Co. Ltd. as a system engineer, Jinan, China. He is currently pursuing the Ph.D. degree with the School of Electrical Engineering, Shandong University, Jinan, China. His research interests include power electronics, renewable energy, and multiagent-based smart microgrids.

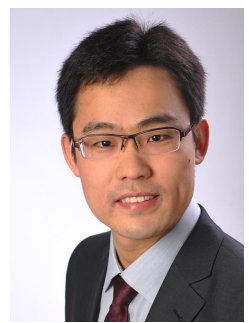

Zhenbin Zhang (Senior Member, IEEE), was born in Shandong, China, in 1984. He received the Ph.D. degree at the Institute for Electrical Drive Systems and Power Electronics (EAL), Technical University of Munich (TUM), Germany, with "summa cum laude". From 2016 to 2017, he worked as a Research Fellow and the group-leader for "Modern control strategies for electrical drives" group in EAL of TUM. Since 2017, he has been a full professor and lab.-director at Shandong Uni., Jinan, China. Since 2020, he has been appointed as the director of International Center for Intelligent Energy and Power Conversion Systems (IEPCS) at Shandong Uni.

Dr. Zhang received the VDE-Award, Suedbayern, Germany, in 2017. He is an Associate Editor for IEEE Trans. Ind. Electron. His research interests include power electronics and electrical drives, sustainable energy systems, smart- and micro-grid.

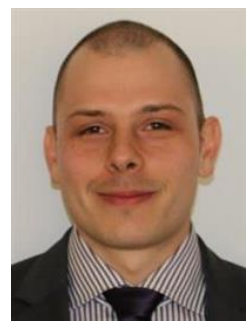

Tomislav Dragičević (Senior Member, IEEE), received the M.Sc. and Industrial Ph.D. degrees in electrical engineering from the Faculty of Electrical Engineering, University of Zagreb, Zagreb, Croatia, in 2009 and 2013 , respectively.

From 2013 to 2016, he has been a Post-Doctoral Researcher with Aalborg University, Aalborg, Denmark, where he was an Associate Professor from 2016 to 2020. Since 2020, he has been a Professor with the Technical University of Denmark, Lyngby, Denmark. He made a guest professor stay at the University of Nottingham, Nottingham, U.K., during spring/summer of 2018. His research interests include the application of advanced control, optimization, and artificial intelligence inspired techniques to provide innovative and effective solutions to emerging challenges in design, control, and cybersecurity of power electronics intensive electrical distributions systems and microgrids. $\mathrm{He}$ has authored and coauthored more than 230 technical publications (more than 100 of them are published in international journals, mostly in IEEE), eight book chapters, and a book in his fields of interest.

Dr. Dragičević was a recipient of the Končar Prize for the Best Industrial $\mathrm{Ph} . \mathrm{D}$. Thesis in Croatia and the Robert Mayer Energy Conservation Award and the winner of an Alexander von Humboldt Fellowship for experienced researchers. He also serves as an Associate Editor for the IEEE Transactions on Industrial Electronics, the IEEE Transactions on Power Electronics, the IEEE Journal of Emerging and Selected Topics in Power Electronics, and the IEEE Industrial Electronics Magazine.

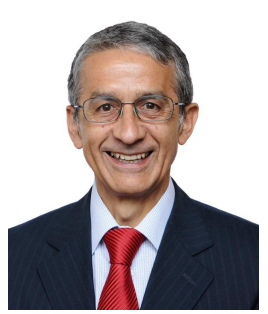

Jose Rodriguez (Fellow, IEEE), Jose Rodriguez (Fellow, IEEE) received the Engineering degree from the Universidad Tecnica Federico Santa Maria, Valparaiso, Chile, in 1977, and the Dr.-Ing. degree from the University of Erlangen, Erlangen, Germany, in 1985 , both in electrical engineering.

Since 1977, he has been a Full Professor and President with the Department of Electronics Engineering, Universidad Tecnica Federico Santa Maria, Valparaiso, Chile. Since 2015, he has been the President with Universidad Andres Bello, Santiago, Chile, where he has been a Full Professor since 2019. He has coauthored two books, several book chapters and more than 400 journal and conference papers. His main research interests include multilevel inverters, new converter topologies, control of power converters, and adjustable-speed drives.

Dr. Rodriguez was a recipient of a number of best paper awards from journals of the IEEE. He is member of the Chilean Academy of Engineering. In 2014, he was the recipient of the National Award of Applied Sciences and Technology from the Government of Chile. In 2015, he was the recipient of the Eugene Mittelmann Award from the Industrial Electronics Society of the IEEE. From 2014 to 2019, he has been included in the list of Highly Cited Researchers published by Web of Science. 